\title{
FLOW PAST A CYLINDER: SHEAR LAYER INSTABILITY AND DRAG CRISIS
}

\author{
S. P. Singh, S. Mittal \\ Department of Aerospace Engineering \\ Indian Institute of Technology \\ Kanpur, UP 208016 \\ India \\ smittal@iitk.ac.in
}

\begin{abstract}
Flow past a circular cylinder for $R e=100$ to $10^{7}$ is studied numerically by solving the unsteady incompressible two-dimensional Navier-Stokes equations via a stabilized finite element formulation. It is well known that beyond $R e \sim 200$ the flow develops significant three dimensional features. Therefore, two dimensional computations are expected to fall well short of predicting the flow accurately at high $R e$. It is fairly well accepted that the shear layer instability is primarily a two dimensional phenomenon. The frequency of the shear layer vortices, from the present computations, agree quite well with the $R e^{0.67}$ variation observed by other researchers from experimental measurements. The main objective of this paper is to investigate a possible relationship between the drag crisis (sudden loss of drag at $R e \sim 2 \times 10^{5}$ ) and the instability of the separated shear layer. As Re is increased the transition point of shear layer, beyond which it is unstable, moves upstream. At the critical Reynolds number the transition point is located very close to the point of flow separation. As a result, the shear layer eddies cause mixing of the flow in the boundary layer. This energizes the boundary layer and leads to its reattachment. The delay in flow separation is associated with narrowing of wake, increase in Reynolds shear stress near the shoulder of the cylinder and a significant reduction in the drag and base suction coefficients. The spatial and temporal power spectra for the kinetic energy of the $R e=10^{6}$ flow are computed. As in two dimensional isotropic turbulence, $E(k)$ varies as $k^{-5 / 3}$ for wavenumbers higher than energy injection scale and as $k^{-3}$ for lower wavenumbers. The present computations suggest that the shear layer vortices play a major role in the transition of boundary layer from laminar to turbulent state.
\end{abstract}


Keywords: vortex shedding, cylinder, drag crisis, shear layer instability, unsteady flow

\section{Introduction}

The flow past a circular cylinder is associated with various instabilities. These instabilities involve the wake, separated shear layer and boundary layer. Williamson ${ }^{1}$ has given a comprehensive description of the flow phenomena at different Reynolds numbers $(R e)$. Upto $R e \sim 47$, the flow is steady with two symmetric vortices on each side of the wake center line. The first wake instability, manifestation of a Hopf bifurcation, occurs at $R e \sim 47$. For $R e>47$, although it remains laminar, the flow becomes unsteady and asymmetric. Von Karman vortex shedding is observed for slightly larger $R e$. At $R e \sim 190$, three-dimensional instabilities, such as formation of vortex loops, deformation of primary vortices and stream wise and span wise vortices, appear in wake. The wake flow undergoes a series of complex three-dimensional instabilities, eventually making it turbulent. Beyond a certain critical Re, the shear layer separating from the upper and lower surface of the cylinder, starts becoming unstable via the Kelvin-Helmholtz mode of instability. The transition point, beyond which the separated layer becomes unstable, moves upstream with increase in $R e$. At $R e \sim 2 \times 10^{5}$, the boundary layer on the cylinder surface undergoes a transition from laminar to turbulent. This transition leads to a delay of the separation of flow from the cylinder surface causing a substantial reduction in the drag force that the cylinder experiences. This is often referred to as Drag crisis.

Bloor $^{2}$ observed the shear layer instability for $R e=1300$ and above. The unstable flow structures were referred to as "transition wave" and identified with Tollmein-Schlichting waves. The ratio of the frequencies of transition waves and primary vortex shedding was found to be proportional to $R e^{0.5}$. Gerrard ${ }^{3}$ observed the shear layer instability at $R e=350$ and higher. Wei and Smith ${ }^{4}$ observed the presence of secondary vortices, similar to transition waves, for $1200 \leq R e \leq 11,000$. The ratio of frequencies associated with the shedding of secondary and primary vortices was found to vary, approximately, as $R e^{0.87}$. Kourta et al. ${ }^{5}$ observed the non-dimensional shedding frequency of the secondary vortices directly proportional to $R e^{0.5}$ for $2000<R e<16,000$. Unal and Rockwell ${ }^{6}$ reported an experimental study of vortex shedding from a circular cylinder for $440 \leq R e \leq 5040$. According to their flow visualization 
experiments, the shear layer instability starts becoming evident at $R e=1900$. Braza et al. ${ }^{7}$ showed the development of Tollmein-Schlichting transition waves in the separated shear layer via two dimensional computations for $2000 \leq R e \leq 10,000$. They concluded that the origin of the shear-layer instability, which leads to mixing layer eddies, is predominantly two-dimensional. The ratio of the computed frequencies of the transition waves and vortex shedding agrees with experimental data of Bloor ${ }^{2}$ and $\mathrm{Kourta}^{5}$ and is quite close to experimental works of Wei and Smith. ${ }^{4}$

Prasad and Williamson ${ }^{8}$ found that the spanwise end conditions, which control the primary mode of vortex shedding, also affect the shear layer instability. For end conditions that result in parallel mode of shedding, shear layer instability starts at $R e \approx 1200$ while this value is 2600 for the end conditions that lead to the oblique shedding mode. The normalized shear layer frequency, equivalent to the non-dimensional shedding frequency of the secondary vortices as used by Wei and Smith, ${ }^{4}$ follows the $R e^{0.67}$ power law. In fact, Prasad and Williamson ${ }^{8}$ plotted not only their own data but also that from all the previous investigators and found that the $R e^{0.67}$ power law gives a much closer fit than the $R e^{0.5}$ law proposed earlier, from approximate analysis. In that sense, the $R e^{0.67}$ power law represents almost all the experimental data that exists. They also observed that the shear layer fluctuations are intermittent and become stronger with increase in Re. Recent computations by Mittal" for a cylinder, with a "slip" splitter plate occupying the wake center line, show that the shear layer instability can be observed for $R e=300$. It was observed that the slip splitter plate annihilates the primary wake instability and, therefore, the shear layer instability can be clearly observed in such a set-up.

Depending on the free stream turbulence and surface roughness, beyond a certain critical $R e$, the boundary layer on the cylinder becomes turbulent. ${ }^{10,11}$ It is accompanied with a significant reduction in drag and is often referred to as drag crisis. It also results in an increase in the base pressure coefficient. The drag coefficient for the subcritical flow is $\sim 1.2$ and it reduces to $\sim 0.3$ for the supercritical flow. For the transcritical flow, ${ }^{10}$ it again increases to $\sim 0.7$. Achenbach and Heinecke ${ }^{11}$ presented the variation of $R e_{c}$ for various values of the surface roughness parameter. For a smooth cylinder, $R e_{c}$ is approximately equal to $3 \times 10^{5}$. With increase in surface roughness, the $R e_{c}$ decreases and the mean drag coefficient at $R e_{c}$ 
increases. Roshko ${ }^{10}$ observed, from experiments, that for sub-critical conditions the boundary layer separation is laminar. As $R e$ is increased, beyond the critical value, transition in boundary layer moves ahead of the laminar separation point. The turbulent boundary layer can withstand a greater pressure rise and delays separation.

Selvam $^{12}$ presented his results for two dimensional Large Eddy Simulation (LES) for flow past a cylinder. The reduction in the drag coefficient was observed but not to the same extent as indicated by the measurements. Further, the reduction was achieved only when the Van Driest damping factor is utilized at the wall. Tamura et al. ${ }^{13}$ carried out computations without any turbulence model with a third order upwind finite difference scheme in two and three dimensions. Their computations have been able to predict drag crisis for certain grids. However, on increasing the number of grid points, the drag coefficient at $R e=10^{6}$ increases significantly.

Cantwell \& Coles $^{14}$ measured various quantities relevant to the Reynolds stresses for the $R e=1.4 \times 10^{5}$ flow corresponding to the high subcritical state. In such a flow large coherent eddies can be studied in their natural state. These eddies are turbulent line vortices which are produced and shed in an essentially regular manner. Except for some dispersion, the vortices are not subject to interactions that might obscure their identity. Reynolds stress receives contribution from, both, the random and periodic motion of the flow. They observed that the contribution of the random turbulent fluctuations, to the Reynolds stress, is much larger than that from the organized large eddies. Periodic stress patterns show a strong symmetry with respect to the wake centerline and remarkable indifference to local turbulence.

In the present study, two-dimensional flow past a circular cylinder is simulated for $R e=$ $100-10^{7}$ using a stabilized finite element formulation. It is well known that beyond $R e \sim 190$ the flow ceases to be two-dimensional. In that sense, the present two dimensional computations are expected to fall well short of accurately predicting the various quantities associated with flows at high Re. The objective of the present work is to investigate a possible connection between the shear layer instability and drag-crisis. It has been reported in the literature ${ }^{7,1}$ that the shear layer instability is primarily two dimensional. A very fine finite element mesh, that is capable of resolving the boundary layer at the cylinder surface and the flow structures associated with the shear layer vortices, is utilized. Computations are carried out at various Re to study 
the shear layer instability. The variation of the frequency of the shear layer vortices with $R e$ is compared to the trends from experimental studies. Good match between the present and published results is observed. It is found that the present computations are able to reproduce the significant drop in the drag coefficient at the critical $R e$. The results indicate that the shear layer instability plays an important role in this phenomenon. In this paper a possible mechanism for the phenomenon is discussed. For high subcritical flow the transition point, beyond which the separated shear layer is unstable, is located shortly downstream of the point of laminar separation of the boundary layer from the cylinder surface. At $R e_{c}$ the transition point moves very close to the separation point and causes local remixing in the boundary layer leading to reattachment of flow.

Direct numerical simulation (DNS) of flows at large $R e$ is not possible owing to the heavy demands on computational resources it places. Beaudan and Moin, ${ }^{15}$ have observed that computations with the Reynolds averaged equations pose great difficulty in predicting the mean forces on the cylinder and the near-wake mean flow statistics. Large eddy simulation (LES), in conjunction with a suitable turbulence model, is a viable proposition. Kravchenko \& Moin ${ }^{16}$ carried out LES for the $R e=3900$ flow past a cylinder using a high order accurate scheme. Good match is seen between their computational results and experiments. They also simulated the flow without the subgrid scale model. No significant difference is found in the mean-velocity profiles from the two cases. However, slower decay of energy at large wave-numbers for the simulations without subgrid model is observed in one-dimensional spectrum of velocity distribution at a downstream location. Mittal and $\operatorname{Moin}^{17}$ reported that the numerical viscosity removes substantial energy from the high wave number regime for higher order upwind-biased finite difference schemes. They also observed that a central difference scheme does not have such problem but it poses additional difficulties related to high dispersion errors. Akin, Tezduyar, Ungor \& Mittal $^{18}$ have shown that the numerical viscosity generated by the stabilization terms for the finite element formulation with bilinear interpolation functions is much higher than the eddy viscosity generated by Smagorinsky turbulence model except in regions very close to the cylinder. In the present study, computations are carried out with and without a Smagorinsky model for flow past a cylinder at $R e=10^{6}$. The mean profiles as well as the fluctuating quantities are compared. It is found that the results from the two sets of computations are 
almost indistinguishable. Therefore, for the computations in this paper, no turbulence model is employed.

The governing equations for the fluid flow are the incompressible Navier-Stokes equations. They are solved via a stabilized finite element formulation in the primitive variables. Equal-inorder linear basis functions for velocity and pressure are used and a 3 point Gaussian quadrature is employed for numerical integration. The non-linear equation systems resulting from the finite element discretization of the flow equations are solved using he Generalized Minimal RESidual (GMRES) technique in conjunction with diagonal preconditioners.

\section{Governing Equations}

Let $\Omega \subset R^{n_{s d}}$ and $(0, T)$ be the spatial and temporal domains respectively, where $n_{s d}$ is the number of space dimensions, and $\Gamma$ denote the boundary $\Omega$. The spatial and temporal coordinates are denoted by $\mathbf{x}$ and $t$. The Navier-Stokes equations governing incompressible fluid flow are

$$
\begin{array}{rr}
\rho\left(\frac{\partial \mathbf{u}}{\partial t}+\mathbf{u} \cdot \nabla \mathbf{u}-\mathbf{f}\right)-\nabla \cdot \sigma=0 & \text { on } \Omega \text { for }(0, T), \\
\nabla \cdot \mathbf{u}=0 & \text { on } \Omega \text { for }(0, T) .
\end{array}
$$

Hence, $\rho, \mathbf{u}, \mathbf{f}$ and $\sigma$ are the density, velocity, body force and the stress tensor, respectively. The stress tensor is written as the sum of its isotropic and deviatoric parts :

$$
\sigma=-p \mathbf{I}+\mathbf{T}, \mathbf{T}=2 \mu \epsilon(\mathbf{u}), \epsilon(\mathbf{u})=\frac{1}{2}\left((\nabla \mathbf{u})+(\nabla \mathbf{u})^{T}\right)
$$

where $\mathrm{p}$ and $\mu$ are the pressure and coefficient of dynamic viscosity, respectively. Both the Dirichlet and Neumann type boundary conditions are accounted for, represented as

$$
\mathbf{u}=\mathbf{g} \text { on } \Gamma_{g}, \mathbf{n} \cdot \sigma=\mathbf{h} \text { on } \Gamma_{h},
$$

where, $\Gamma_{g}$ and $\Gamma_{h}$ are complementary subsets of the boundary $\Gamma$. The initial condition on the velocity is specified on $\Omega$ :

$$
\mathbf{u}(\mathbf{x}, 0)=\mathbf{u}_{0} \text { on } \Omega,
$$

where, $\mathbf{u}_{0}$ is divergence free. 
In addition to the mean flow, it is useful to study the Reynolds stresses $\left(\overline{u^{\prime} u^{\prime}}, \overline{v^{\prime} v^{\prime}}\right.$ and $\left.\overline{u^{\prime} v^{\prime}}\right)$. Here, $u^{\prime}$ and $v^{\prime}$ are the fluctuations in the components of the velocity field as defined below, following the decomposition proposed by Hussain and Reynolds ${ }^{19}$ :

$$
\mathbf{u}(\mathbf{x}, t)=\overline{\mathbf{u}}(\mathbf{x})+\mathbf{u}^{\prime}(\mathbf{x}, t) .
$$

Here $\overline{\mathbf{u}}(\mathbf{x})$ is the mean velocity field, and $\mathbf{u}^{\prime}(\mathbf{x}, t)$ is the unsteady part of the velocity with contributions from organized wave and turbulence.

\section{Finite Element Formulation}

Consider a finite element discretization of $\Omega$ into sub domains $\Omega^{e}, e=1,2, \ldots, n_{e l}$, where $n_{e l}$ is the number of elements. Based on this discretization, for velocity and pressure we define the finite element trial function spaces $\mathcal{S}_{\mathbf{u}}{ }^{h}$ and $\mathcal{S}_{p}^{h}$, and weighting function spaces $\mathcal{V}_{\mathbf{u}}{ }^{h}$ and $\mathcal{V}_{p}^{h}$. These function spaces are selected, by taking the Dirichlet boundary conditions into account, as subsets of $\left[\mathbf{H}^{1 h}(\Omega)\right]^{n_{s d}}$ and $\mathbf{H}^{1 h}(\Omega)$, where $\mathbf{H}^{1 h}(\Omega)$ is the finite dimensional function space over $\Omega$. The stabilized finite element formulation of equations 1 and 2 is written as follows: find $\mathbf{u}^{h} \in \mathcal{S}_{\mathbf{u}}{ }^{h}$ and $p^{h} \in \mathcal{S}_{p}^{h}$ such that $\forall \mathbf{w}^{h} \in \mathcal{V}_{\mathbf{u}}{ }^{h}, q^{h} \in \mathcal{V}_{p}^{h}$

$$
\begin{aligned}
\int_{\Omega} \mathbf{w}^{h} \cdot \rho\left(\frac{\partial \mathbf{u}^{h}}{\partial t}+\mathbf{u}^{h} \cdot \nabla \mathbf{u}^{h}-\mathbf{f}\right) & d \Omega+\int_{\Omega} \epsilon\left(\mathbf{w}^{h}\right): \sigma\left(p^{h}, \mathbf{u}^{h}\right) d \Omega+\int_{\Omega} q^{h} \nabla \cdot \mathbf{u}^{h} d \Omega \\
& +\sum_{e=1}^{n_{e l}} \int_{\Omega^{e}} \frac{1}{\rho}\left(\tau_{S U P G} \rho \mathbf{u}^{h} \cdot \nabla \mathbf{w}^{h}+\tau_{P S P G} \nabla \mathbf{q}^{h}\right) . \\
& {\left[\rho\left(\frac{\partial \mathbf{u}^{h}}{\partial t}+\mathbf{u}^{h} \cdot \nabla \mathbf{u}^{h}-\mathbf{f}\right)-\nabla \cdot \sigma\left(p^{h}, \mathbf{u}^{h}\right)\right] d \Omega^{e} } \\
& +\sum_{e=1}^{n_{e l}} \int_{\Omega^{e}} \delta \nabla \cdot \mathbf{w}^{h} \rho \nabla \cdot \mathbf{u}^{h} d \Omega^{e}=\int_{\Gamma_{h}} \mathbf{w}^{h} \cdot \mathbf{h}^{h} d \Gamma
\end{aligned}
$$

In the variational formulation given by equation 7 , the first three terms and the right hand side constitute the Galerkin formulation of the problem. The first series of element level integrals are the SUPG and PSPG stabilization terms added to the variational formulations. In the current formulation $\tau_{P S P G}$ is the same as $\tau_{S U P G}$ and is given as

$$
\tau=\left(\left(\frac{2\left\|\mathbf{u}^{h}\right\|}{h}\right)^{2}+\left(\frac{4 \nu}{h^{2}}\right)^{2}\right)^{-\frac{1}{2}}
$$

The second series of element level integrals are added to the formulation for numerical stability at high Reynolds numbers. This is a least squares terms based on the continuity equation. The 
coefficient $\delta$ is defined as

$$
\delta=\frac{h}{2}\left\|\mathbf{u}^{h}\right\| z
$$

where,

$$
z=\left\{\begin{array}{cc}
\left(\frac{R e_{u}}{3}\right) & R e_{u} \leq 3 \\
1 & R e_{u}>3
\end{array}\right.
$$

and $R e_{u}$ is the cell Reynolds number. Both stabilization terms are weighted residuals, and therefore maintain the consistency of the formulation. $h$ is the element length and various definitions have been used by researchers in the past. Mittal ${ }^{20}$ conducted a systematic numerical study to investigate the effect of high aspect ratio elements on the performance of the finite element formulation for three commonly used definitions of $h$. The one which results in the least sensitivity of the computed flow to the element aspect ratio has been used for computations in the present work. According to this definition, the element length is equal to the minimum edge length of a triangular (3 noded) element.

The time discretization of the variational formulation is done via the generalized trapezoidal rule. For unsteady computations, the relevant parameter is set to give second order accuracy in time. Equal in order basis functions for velocity and pressure (the P1P1 element) are used and a 3 point quadrature is employed for numerical integration. The non-linear equation systems resulting from the finite element discretization of the flow equations are solved using the Generalized Minimal Residual (GMRES) technique ${ }^{21}$ in conjunction with a diagonal preconditioner. A matrix free version of the GMRES algorithm is utilized to reduce the memory requirement. In this procedure, the result of matrix vector products in the GMRES algorithm is direct computed which avoids the explicit formation and storage of the element level matrices.

\section{Results and Discussions}

A cylinder, of diameter $D$, is placed in a domain whose outer boundary is a rectangle. The center of the cylinder is located at the origin of the coordinate system. The free-stream flow is along the $x$ axis. The Reynolds number, $R e$, is based on the diameter of the cylinder, freestream velocity and viscosity of the fluid. In all the figures in this paper, the shading in gray scale represents the magnitude of the associated flow quantity. While the white shade shows a low value, the darker shade represents a higher value. The contour lines in black color indicate 
a positive value of the variable and a negative value is shown by lines in white color.

\section{A. Boundary conditions}

Free-stream values are assigned for the velocity at the upstream boundary. No slip condition for the velocity is applied on the cylinder boundary. At the downstream boundary a Neumann type boundary condition for the velocity is specified that corresponds to zero viscous stress vector. On the upper and lower boundaries the component of stress vector along these boundaries and the velocity normal to them are assigned zero values.

\section{B. Finite element mesh}

The finite element mesh consists of two parts: a structured grid close to the cylinder and an unstructured mesh in the remaining domain. The structured mesh allows for having adequate control on the resolution of the flow in the boundary layer. It consists of $N_{t}$ nodes in the circumferential direction. The radial thickness of the first layer of elements on the cylinder boundary is denoted by $h_{r}^{1}$. The unstructured mesh is generated via the Delaunay's triangulation technique. This kind of a hybrid mesh is useful in handling complex geometries by providing adequate resolution close to the body without requiring the same distribution of grid points in the remaining domain. This results in significant saving of computational resources as opposed to computations on a structured mesh with similar resolution close to the body.

Given in Table 1 are the details of the various meshes used in the present study. Figure 1 shows the close-up views of a typical mesh around the cylinder. Most of the computations in this paper have been done with mesh $M 4$ which consists of 47, 011 nodes and 93, 574 elements. The time-averaged drag-coefficient $\left(\bar{C}_{d}\right)$ for $R e=10^{6}$ obtained with the mesh $M 4$ is 0.591 . To check the convergence of the computations with respect to spatial resolution, the flow is also computed with a more refined mesh, M5. Mesh M5 consists of 116, 166 nodes and 231, 484 elements. The value of $\left(\bar{C}_{d}\right)$ with mesh $M 5$ is 0.607 . Other mean and fluctuating quantities also show a good match between the results from the two meshes. This establishes the adequacy of mesh M4 to resolve most of the large scale structures of the flow in this range of $R e$. 


\section{Large Eddy Simulation vs. model free calculations}

It is not possible, with the present computational resources, to conduct DNS for the range of $R e$ that are being attempted in this study. LES in conjunction with a suitable sub-grid scale model seems like a viable proposition. A LES with a constant coefficient Smagorinsky model was attempted for $R e=10^{6}$. The dynamic coefficient of viscosity, $\mu$, is locally modified using a Smagorinsky turbulence model as $\mu_{\text {effective }}=\mu+\rho\left(C h_{e}\right)^{2} \sqrt{2 \epsilon(\mathbf{u}): \epsilon(\mathbf{u})}$. Here $C$ is a constant equal to 0.1 and $h_{e}$ is the element length scale. Mesh M4, with 47,011 nodes and 93, 574 elements, is employed for the computations. Computations are also carried out without the turbulence model. Figure 2 shows the time averaged vorticity, pressure, $\overline{u^{\prime} u^{\prime}}, \overline{v^{\prime} v^{\prime}}$ and $\overline{u^{\prime} v^{\prime}}$ fields for the model free calculations (first column) and LES (second column). It can be observed that the results from the two simulations are very comparable. Other quantities, for example, the time averaged drag and base pressure coefficients are also very comparable. They are shown in later figures in the paper. This is consistent with the observations of Akin, Tezduyar, Ungor \& Mittal ${ }^{18}$ who found that the numerical diffusion due to the stabilization terms in the finite element formulation, with bilinear interpolation functions, is much higher than the eddy viscosity generated by Smagorinsky turbulence model except in regions very close to the cylinder. In view of these observations, all further simulations are carried out without any turbulence model. It is quite possible that the situation may change for three dimensional simulations or with computations employing higher order interpolation functions. These issues are under investigation.

We wish to reiterate here that 2D LES by no means captures all the details of an inherently three-dimensional flow. The attempt in this paper is to investigate the possibility of a connection between the shear layer instability (which is known to be two-dimensional) and drag-crisis and not to model/resolve the 3D effects. In addition to LES, there are other approaches that have shown promise in solving 3D unsteady flows. For example, the interested reader may see the work by Travin et al. ${ }^{22}$ who have simulated flow past a circular cylinder via Detached Eddy Simulation for laminar and turbulent separation. Reynolds numbers chosen for laminar separation are $5.0 \times 10^{4}$ and $1.4 \times 10^{5}$ while those for turbulent separation are $1.4 \times 10^{5}$ and $3.0 \times 10^{6}$. The results of turbulent separation cases, i.e., drag, skin friction, shedding frequency 
and pressure match well with the experimental results.

\section{General overview of the flow}

Figures 3 and 4 show the variation, with $R e$, of the time-averaged drag and base suction coefficient, respectively. Data from computations on various finite element grids has been shown along with experimental results from other researchers. It is observed that the values from present computations match well with the results from experiments for $R e<200$. For example, the Strouhal number (St: non-dimensional vortex shedding frequency based on the dominant frequency in the time variation of lift coefficient) for $R e=100$, from the present two dimensional computations, is 0.1643 . Williamson ${ }^{23}$ measured $S t=0.1648$ for parallel shedding and the value reported by Kravchenko, Moin and Shariff ${ }^{24}$ and Persillon and Braza ${ }^{25}$ from their computations is 0.164 . The amplitude of the lift coefficient from the present computations is 0.319 and it compares well with the value reported by Kravchenko, Moin and Shariff ${ }^{24}(=0.314)$. For $R e=200$, the values for $\bar{C}_{D}$ and $S t$ from the present two-dimensional computations are 1.327 and 0.1947, respectively. The corresponding values from Posdziech and Grundmann ${ }^{26}$ from their computational studies are 1.3132 and 0.1944 . The experimentally measured value for $S t$ is $0.196 .^{27}$ Again, the comparison with the present results is excellent.

Beyond $R e \sim 180$ the wake flow undergoes three-dimensional transitional instabilities. Therefore, for $R e>200$, the drag and base suction coefficient and Strouhal number are over predicted by two dimensional computations. This observation was made by $\mathrm{Mittal}^{28}$ for two and and three dimensional computations for the $R e=300$ and 1000 flow past a cylinder. For example, the three dimensional computations for the $R e=300$ flow with slip side walls by Mittal, ${ }^{28}$ using a very similar finite element formulation as in this work, results in $S t=0.203$. The present two dimensional computations, as expected, result in a slightly larger value of St $(=0.208)$. Kravchenko, Moin and Shariff, ${ }^{24}$ from their three dimensional computations, observed $S t=0.203$ and $\bar{C}_{D}=1.28$. The corresponding experimental values from Williamson ${ }^{1}$ are 0.203 and 1.22 . The mean drag coefficient from the present two dimensional computations for $R e=300$ is 1.357. Mittal and Balachander ${ }^{29}$ have suggested that the higher value of the drag coefficient for the $2 \mathrm{D}$ simulations is caused due to higher level of Reynolds stresses resulting in a shorter formation length behind the bluff body. Detailed results for the flow at 
low $R e(100<R e<1000)$ with the same finite element formulation as in this work, and their comparison with experiments, can be found in our earlier papers . . $^{30,31,28}$

It is clear from Figures 3 and 4 that as the three dimensional features in the flow become increasingly important, the two-dimensional computations over predict the mean drag and base suction coefficient for $2 \times 10^{3}<R e<3.2 \times 10^{4}$. Three very interesting observations can be made from these figures. First, consider the variation of $-C_{P B}$ with $R e$ (Figure 4 ) in the regime where the flow is two dimensional. Also shown in the figure is a straight line that passes through the data points for $R e<200$. It can be observed that the results from twodimensional computations for $2 \times 10^{3}<R e<3.2 \times 10^{4}$ lie close to the line extrapolated from the two-dimensional behavior. Also, the difference between the results from two-dimensional calculations and real three dimensional flow increases rapidly with $R e$. A similar observation was made by Mittal and Balachander. ${ }^{29}$ However, their data is for a smaller range of Re. Higher value of base suction results in larger drag force experienced by the cylinder. Consequently, two dimensional computations overpredict the drag coefficient. The second interesting observation is that the present, two dimensional, computations are able to pick up the sudden reduction in drag and base suction coefficient close to the critical Re. This suggests that the drag crisis is largely a two-dimensional phenomenon. Also, at the critical $R e$ and beyond, the two-dimensional computations result in a fairly good prediction of the time-averaged drag coefficient. The third observation from the two figures is regarding the behavior of the flow in the supercritical state. Subsequent to the drag crisis, an increase in, both, the mean drag coefficient and base suction coefficient is observed. This behavior is replicated by the present computations. Another observation that can be made from Figures 3 and 4 is the excellent agreement between the results obtained with computations using different meshes. For example, meshes $M 2$ and M4 give very similar values for the $R e=10^{4}$ flow.

The time averaged vorticity field and Reynolds stress distribution $\left(\overline{u^{\prime} u^{\prime}}, \overline{v^{\prime} v^{\prime}}\right.$ and $\left.\overline{u^{\prime} v^{\prime}}\right)$ for various $R e$ are shown in Figure 5. Narrowing of the wake for the $R e=10^{6}$ flow, compared to flow at lower Re, is clearly observed. Our results indicate that the Reynolds stresses are symmetric about the center axis and the normal components are, in general, larger than the shear components. Similar to the observations of Cantwell \& Coles $^{14}$ and Mittal \& Balachandar, ${ }^{29}$ it is seen that the $\overline{v^{\prime} v^{\prime}}$ field achieves a peak along the center axis while the peaks in $\overline{u^{\prime} u^{\prime}}$ and 
$\overline{u^{\prime} v^{\prime}}$ are achieved off the flow axis, within the wake bubble. At $R e=100$ the Reynolds stresses close to the cylinder are very small. With increase in $R e$ their magnitude, close to the cylinder especially in the region of separated shear layer, increases. This suggests an increased unsteady activity, for higher $R e$, in that region of the flow.

\section{E. Shear layer instability}

Figure 6 shows the instantaneous vorticity field for various $R e$. For the $R e=100 \& 200$ flow the wake is very organized and the regular von Karman vortex street is observed. At higher $R e$, the separated shear layer becomes unstable and smaller vortices form as a result of this instability. The fluctuations in the velocity field due to the shear layer instability are intermittent and their strength increases with $R e$. In addition, the point at which the separated shear layer becomes unstable moves upstream with an increase in $R e$. Some of these features can be observed from Figure 6. As is observed for the time averaged flow, the narrowing of the wake for the $R e=10^{6}$ flow is seen here, as well.

The time histories of the drag and lift coefficients for various Re are shown in Figure 7. The aerodynamic coefficients exhibit a very organized behavior for low $R e$. For $R e=100$ and 200, a single frequency, corresponding to the vortex shedding frequency $\left(f_{K}\right)$, is observed. The onset of shear layer instability modifies this variation. In addition to $f_{K}$ a smaller frequency, $f_{S L}$, that corresponds to the oscillations due to the shear layer vortices, sets in. It is seen from the time variation of the lift coefficient at $R e=10^{4}$ (Figure 7 ) that the shear layer instability is intermittent. It is more regular at larger $R e$.

Prasad and Williamson ${ }^{8}$ studied the shear layer instability via laboratory experiments. They suggested two techniques for determining the shear layer frequency $\left(f_{S L}\right)$. In the first technique, $f_{S L}$ corresponds to the maximum in the broad-band peak of the long-time-averaged velocity spectra. Since the shear-layer instability occurs intermittently, long-time-averaged velocity spectra appears reduced in amplitude. In the second method, $f_{S L}$ is measured from the period of shear-layer fluctuations using time traces. A statistically significant sample is chosen to estimate a reasonable value. A histogram between the percentage of total number of shear-layer cycles and frequency is utilized to select $f_{S L}$. It corresponds to that frequency which has the most prominent bin in the histogram. Both techniques result in very similar results. 
Figure 8 shows the ratio of shear layer and primary vortex shedding frequencies $\left(f_{S L} / f_{K}\right)$ from the present computations for various $R e$ and their comparison with the measurements from other researchers. The "histogram" technique proposed by Prasad and Williamson ${ }^{8}$ has been utilized in the present work. Good match is observed with the $R e^{0.67}$ variation for $R e$ upto $2 \times 10^{5}$. This confirms the observation of Braza, Chassaing \& Ha Minh ${ }^{7}$ that the origin of the shear layer instability and the subsequent development of small scale vortices is essentially a two dimensional phenomenon. The agreement at $R e=10^{6}$ is poor. This is because at this $R e$, the transition point at which the separated shear layer becomes unstable reaches the cylinder surface. Consequently, the generation and time evolution of the shear layer vortices is influenced by the cylinder surface and the boundary layer on it. As a result the separated boundary layer ceases to behave as a free shear layer. It is clear from this plot that $f_{S L}$ increases with $R e$. This is consistent with the vorticity field shown in Figure 6 where the number of shear layer vortices generated/released per unit time increases with $R e$.

\section{F. Drag crisis and shear layer instability}

Figure 9 shows close-up views of the instantaneous vorticity field at various $R e$ near the upper and lower surfaces of the cylinder. The reduction in the boundary layer thickness with the increase in $R e$ has been adequately resolved by an appropriate finite element mesh near the cylinder surface. The instability of the separated shear layer and its interaction with the boundary layer on the cylinder surface can be observed from this figure. For the $R e=3.2 \times 10^{4}$ flow the transition point, for the onset of shear layer instability, is located fairly downstream of the cylinder. It moves upstream, towards the cylinder, with increase in $R e$. At $R e=10^{5}$ the shear layer becomes unstable shortly downstream of the point of flow separation from the cylinder surface. At $R e=10^{6}$ the point of instability reaches very close to the un-separated boundary layer on the surface of the cylinder. As a result of these eddies the boundary layer experiences mixing with the outer flow causing reattachment of flow and delay of separation. This is associated with narrowing of wake and significant reduction in drag and base suction coefficients. At $R e=10^{7}$, the instability of the shear layer propagates further upstream and causes mixing of a significant portion of the boundary layer prior to its eventual separation. The mixing of flow results in a significant increase in the Reynolds stresses in this region. Figure 10 
shows the Reynolds shear stress near the cylinder surface in the region of flow separation. The time averaging for $\overline{u^{\prime} v^{\prime}}$ shown in this figure has been carried out for one shear layer cycle for each case.

These results suggest that the transition of the boundary layer, on the surface of cylinder, is initiated by the instability of the separated shear layer. For the sub-critical flow, the instability of the separated shear layer commences sufficiently downstream of the point of separation. At the critical Reynolds number, the separated laminar boundary layer experiences significant mixing shortly downstream of its separation, due to the eddies generated by the shear layer vortices. This marks the transition of the separated boundary layer from laminar to turbulent and causes the flow to reattach. The eventual separation of the re-attached flow occurs at a much downstream location and results in drag-crisis. For higher Re the shear layer instability moves upstream and so does the transition point of the boundary layer. Eventually, the entire boundary layer on the cylinder surface becomes turbulent. This results in an increase in the drag experienced by the cylinder due to the increased skin-friction on its surface. This can also be observed from Figure 3 which shows the variation in the time-averaged drag coefficient for various $R e$. As is observed from experimental data, the drag coefficient for $R e=10^{7}$ is larger than that at $R e=10^{6}$.

Shown in Figure 11 is the instantaneous vorticity field for the $R e=10^{6}$ flow along with the velocity profiles (in wall coordinates) at certain locations. Also shown, in broken line, is the velocity profile in the viscous sub-layer and log layer for a turbulent boundary layer on a flat plate. These plots indicate that the present simulations are fine enough to resolve the boundary layer and the associated flow structures of similar scale close to the cylinder. The velocity profile at station (a), before the flow separates from the surface of cylinder, matches that for a laminar boundary layer over flat plate. Shortly downstream of the flow separation, the shear layer eddies cause mixing of flow leading to the reattachment of boundary layer. At stations shortly downstream of this phenomenon, the velocity profile looks similar to that of a turbulent boundary layer over a flat plate in adverse pressure gradient. The presence of the log layer is indicative of a possible turbulent boundary layer. In contrast, for the $R e=10^{5}$ flow, the log layer is observed at locations sufficiently downstream of the point of separation after the flow has undergone intense mixing. The present simulations are not fine enough to resolve 
the random turbulent fluctuations. However, it appears that at least the large scale structures that are mainly responsible for the transition of boundary layer are sufficiently resolved in these computations.

Figure 12 shows time averaged pressure coefficient $\left(C_{P}\right)$ distribution on the surface of the cylinder. It is observed that $C_{P}$ at $\theta=0$, the front stagnation point, is more than 1.0 for low $R e$, for example, at $R e=100$. This is consistent with the observations of earlier researchers. With increase in $R e$, the peak suction pressure coefficient $\left(-C_{P}\right)$ increases. As is seen from Figure 4, the base pressure coefficient $\left(C_{P}\right.$ at $\left.\theta=180^{\circ}\right)$ first decreases (achieves a larger negative value) with $R e$ and than increases. Compared to the flow at $R e=10^{5}$, the delay in flow separation for $R e=10^{6}$ results in a higher peak suction, near the shoulder of the cylinder, and a higher base pressure. The higher base pressure results in a lower drag coefficient. It is also seen that for the $R e=10^{6}$ flow, the 2D LES with a Smagorinsky turbulence model results in virtually identical pressure distribution as the model free computations.

The pressure distribution for $R e=10^{5}$ shows a second local suction peak on the surface of the cylinder. This secondary peak occurs beyond the shoulder of the cylinder and points to the presence of a local recirculation zone close to the surface. Figure 13 shows the time averaged streamlines for the flows at $R e=2000,10^{5}$ and $10^{6}$. It is observed that the $R e=2000$ flow is associated with two recirculation zones on each half of the cylinder. However, they are located away from the surface of the cylinder and the speed of flow in these regions is relatively small. Consequently, their effect is not felt by the surface $C_{P}$ distribution. At $R e=10^{5}$, downstream of the separation point, the speed of the flow in the reverse flow region is quite high very close to the surface of the cylinder. This results in a local peak in the suction pressure. At $R e=10^{6}$, it disappears again because of the delay in flow separation. The time-averaged streamlines and pressure distributions suggest that the $R e=10^{5}$ is associated with a recirculatory laminar "bubble" close to the cylinder, immediately downstream of the point of flow separation. At higher $R e$, the shear layer vortices cause the flow to become turbulent and the laminar bubble bursts.

The time averaged flow from the present computations appear symmetric about the $x$ axis for $R e=10^{6}$. The flow at $R e=10^{5}$ clearly shows some asymmetry. Schewe ${ }^{32}$ conducted 
force measurements in a pressurized wind tunnel from subcritical up to transcritical Re, i.e., $2.3 \times 10^{4} \leq R e \leq 7.1 \times 10^{6}$. It was shown in this study that asymmetric flow separation with the generation of non-zero steady lift in critical regime $\left(2.8 \times 10^{5} \leq R e \leq 3.5 \times 10^{5}\right)$, of both the positive and negative sign, is a fundamental phenomenon. This phenomenon is marked by two discontinuous transitions, i.e. drop and jump in $C_{D}$ and $S t$. After the first transition, the flow achieves a bistable asymmetric state consisting of two stable states corresponding to positive and negative lift force. The second transition is characterized by further jump and drop in St and $C_{D}$ and the abrupt disappearance of the steady lift. Bearman ${ }^{33}$ examined the flow around a circular cylinder over the $R e$ range $10^{5}$ to $7.5 \times 10^{5}$. A discontinuity was recorded at $R e \sim$ $3.4 \times 10^{5}$ in the variation of the base pressure coefficient. This is caused by the appearance of a laminar separation bubble forming on only one side of the cylinder. This appearance extends up to $R e=3.8 \times 10^{5}$. At both the ends of the regime, marked by the laminar separation bubble, discontinuous drop and jump in Strouhal number and the drag coefficient, respectively, are observed. Detailed computations close to $R e_{c}$ need to be carried out to further investigate this behavior of the flow.

\section{G. Energy spectra}

In this section results are presented for the energy spectra of the flow. To construct a spectrum of the energy at various spatial scales, the solution obtained from the Navier-Stokes equations over an unstructured mesh is interpolated on a structured mesh. The two-dimensional, discrete Fast Fourier Transform (FFT) of the velocity field is carried out by using the subroutines in the library from the Numerical Algorithm Group (NAG). The temporal spectrum is constructed by sampling the time history of the velocity at a point $(x / D=0.26, y / D=-0.46)$ with respect to the center of the cylinder.

In three-dimensional turbulence, energy injected into a flow system at a low wave number cascades to higher wave numbers via vortex stretching. In this inertial range the structure of energy density $E(k)$ is determined solely by the non-linear interactions while the total energy $\int E(k) d k$ is conserved. In the inertial range $E(k)$ varies as $k^{-5 / 3}$ down to the length scales where viscous effects cause a rapid decay of $E(k)$. In two-dimensional flows, the vortex stretching mechanism is absent. Consequently, both, energy $\left(\int E(k) d k\right)$ and enstrophy (square of $L^{2}$ norm 
of vorticity, $\int_{\Omega}|\omega|^{2} d \Omega$ ) are conserved. This implies that any flow of energy from low to high wave numbers is accompanied with another flux, back from small to larger length scales. This characteristic of two-dimensional turbulence is called inverse cascade. ${ }^{34}$ The enstrophy cascade follows the $k^{-3}$ law. More details on two-dimensional turbulence can be found in Paret \& Tabeling, ${ }^{35}$ Frisch, ${ }^{36}$ Doering \& Gibbon ${ }^{37}$ and Lesieur. ${ }^{38}$

It is well known that even for high subcritical Re the flow in the wake, outside the boundary layer, is turbulent. The spatial power spectra for the $R e=10^{6}$ flow is shown in Figure 14 . The mesh used for the flow computations is M5. The velocity field is interpolated on a structured grid with $3648 \times 1536$ nodes. As expected in two-dimensional isotropic turbulence, both, $k^{-3}$ and $k^{-5 / 3}$ variations of $E(k)$ are observed. The power spectra corresponding to the time history of the velocity at a point in the wake is shown in Figure 15. The frequency in the time domain is non-dimensionalized with the vortex shedding frequency. A similar distribution, as in the spatial spectrum, is observed.

\section{Concluding Remarks}

Flow past a circular cylinder is simulated for $R e=100$ to $10^{7}$ via a stabilized finite element formulation in two dimensions. The computations with and without a sub-grid scale model lead to very similar results. As expected, beyond $R e \sim 200$, the $2 \mathrm{D}$ computations are unable to predict the correct value of drag coefficient. However, the phenomenon of drag-crisis is predicted quite well. For Re $\sim 2000$ and larger, in addition to the primary wake instability, the instability of the separated shear-layer is observed. The mesh close to the cylinder is fine enough to resolve the boundary layer and its interaction with the shear layer instability. It is found that the ratio of the shear layer and vortex shedding frequencies varies as $R e^{0.67}$, in agreement with results from other researchers. The transition point beyond which the separated shear layer becomes unstable moves upstream with $R e$. The unstable shear layer is responsible for the small scale vortices. At a certain critical Reynolds number $\left(R e_{c}\right)$, the transition point moves very close to the separation point leading to an interaction between the boundary layer and the shear layer vortices. These vortices cause mixing of flow in the boundary layer, thereby energizing it and leading to its reattachment. An increase in the Reynolds shear stress near the 
separation point is observed as a result of this vortical activity. The entire boundary layer, till the point of separation, is laminar for $R e<R e_{c}$. At $R e=R e_{c}$ even though a significant part of the boundary layer is laminar, the latter part, beyond the point of reattachment, undergoes a transition to a turbulent state. The flow is associated with narrowing of wake and a significant reduction in the time averaged drag- and base suction coefficients. Beyond $R e_{c}$, the boundary layer achieves a turbulent state well ahead of the flow separation. This leads to increased skin friction and drag coefficient.

The transition of the boundary layer to a turbulent state is also observed from the velocity profiles. These computations highlight a possible mechanism that lead to the transition of the boundary layer flow. The interaction between the shear layer eddies and the boundary layer plays a major role in the transition. The computations also suggest that the genesis and development of the shear layer vortices and their interactions with the boundary layer leading to its transition to a turbulent state is primarily a two dimensional phenomenon. Almost all the features of the flow are captured by the present two dimensional computations, qualitatively. Three-dimensional effects need to be accounted for to obtain good quantitative comparisons with experimental results. Although the present computations do not utilize grids that are fine enough to resolve the random fluctuations associated with transitional flows, yet, most of the flow features are predicted reasonably well. This shows that the large scale flow structures play a primary role in this transition process.

The spatial and temporal energy spectra for the $R e=10^{6}$ flow have been studied. It is found that the energy spectrum exhibits the structure of two-dimensional isotropic homogeneous turbulence. The $k^{-3}$ and $k^{-5 / 3}$ variation of $E(k)$, for $k$ higher and lower than the energy injection scale, respectively, is observed.

\section{Acknowledgment}

Partial support for this work has come from the Department of Science and Technology, India. 


\section{References}

${ }^{1}$ C.H.K. Williamson. Vortex dynamics in the cylinder wake. Annual Review of Fluid Mechanics, 28:477-539, 1996.

${ }^{2}$ M. S. Bloor. The transition to turbulence in the wake of a circular cylinder. Journal of Fluid Mechanics, 19:290, 1964.

${ }^{3}$ J.H. Gerrard. The wakes of cylindrical bluff bodies at low Reynolds number. Philosophical Transactions of the Royal Society of London A, 288:351-382, 1978.

${ }^{4}$ T. Wei and C.R. Smith. Secondary vortices in the the circular cylinders. Journal of Fluid Mechanics, 169:513-533, 1986.

${ }^{5}$ A. Kourta, H.C. Boisson, P. Chassaing, and H. Ha Minh. Nonlinear interaction and the transition to turbulence in the wake of a circular cylinder. Journal of Fluid Mechanics, 181:141-161, 1987.

${ }^{6}$ M.F. Unal and D. Rockwell. On the vortex formation from a cylinder Part 1. The initial instability. Journal of Fluid Mechanics, 190:491-512, 1988.

${ }^{7}$ M. Braza, P. Chassaing, and H. Ha Minh. Prediction of large-scale transition features in the wake of a circular cylinder. Physics of Fluids A, 2:1461-1471, 1990.

${ }^{8}$ A. Prasad and C.H.K. Williamson. The instability of the shear layer separating from a bluff body. Journal of Fluid Mechanics, 333:375-402, 1997.

${ }^{9}$ S. Mittal. Shear layer instability in flow past a cylinder at low Reynolds number. submitted to Physics of Fluids, 2003.

${ }^{10}$ A. Roshko. Experiments on the flow past a circular cylinder at very high Reynolds number. Journal of Fluid Mechanics, 10:345-356, 1961.

${ }^{11}$ E. Achenbach and E. Heinecke. On vortex shedding from smooth and rough cylinders in the range of Reynolds numbers $6 \times 10^{3}$ to $5 \times 10^{6}$. Journal of Fluid Mechanics, 109:239-251, 1981. 
${ }^{12}$ R.P. Selvam. Finite element modeling of flow around a circular cylinder using LES. Journal of Wind Engineering and Industrial Aerodynamics, 67 \& 68:129-139, 1997.

${ }^{13}$ T. Tamura, I. Ohta, and K. Kuwahara. On the reliability of two-dimensional simulation for unsteady flows around a cylinder-type structure. Journal of Wind Engineering and Industrial Aerodynamics, 35:275-298, 1990.

${ }^{14}$ B. Cantwell and D. Coles. An experimental study of entrainment and transport in the turbulent near wake of a circular cylinder. Journal of Fluid Mechanics, 136:321-374, 1983.

${ }^{15}$ P. Beaudan and P. Moin. Numerical experiments on the flow past a circular cylinder at subcritical Reynolds number. Technical Report TF-62, Department of Mechanical Engineering, Stanford University, 1994.

${ }^{16}$ A.G. Kravchenko and P. Moin. Numerical studies of flow over a circular cylinder at $R e_{D}=$ 3900. Physics of Fluids, 12:403-417, 2000.

${ }^{17}$ R. Mittal and P. Moin. Suitability of upwind-biased-finite difference schemes for large-eddy simulation of turbulent flows. AIAA Journal, 35:1415, 1997.

${ }^{18}$ J.E. Akin, T.E. Tezduyar, T.E. Ungor, and S. Mittal. Stabilization parameters and smagorinsky turbulence model. Journal of Applied Mechanics, 12:1-20, 2002.

${ }^{19}$ A.K.M.F. Hussain and W.C. Reynolds. The mechanics of an organized wave in turbulent shear flow. Part 2. Experimental results. Journal of Fluid Mechanics, 54:241-261, 1972.

${ }^{20} \mathrm{~S}$. Mittal. On the performance of high aspect-ratio elements for incompressible flows. Computer Methods in Applied Mechanics and Engineering, 188:269-287, 2000.

${ }^{21}$ Y. Saad. Krylov subspace methods for solving large unsymmetric linear systems. Mathematics of Computation, 37(155):105-126, 1981.

${ }^{22}$ A. Travin, M. Shur, M. Strelets, and P. Spalart. Detached-eddy simulations past a circular cylinder. Flow, Turbulence and Combustion, 63:293-313, 2000.

${ }^{23}$ C.H.K. Williamson. Oblique and parallel modes of vortex shedding in the wake of a circular cylinder at low Reynolds numbers. Journal of Fluid Mechanics, 206:579-627, 1989. 
${ }^{24}$ A.G. Kravchenko, P. Moin, and K. Shariff. B-Spline method and zonal grids for simulations of complex turbulent flows. Journal of Computational Physics, 151:757-789, 1999.

${ }^{25}$ H. Persillon and M. Braza. Physical analysis of the transition to turbulence in the wake of a circular cylinder by three-dimensional Navier-Stokes simulation. Journal of Fluid Mechanics, 365:23-88, 1998.

${ }^{26}$ O. Posdziech and R. Grundmann. Numerical simulation of the flow around an infinitely long circular cylinder in the transition regime. Theoretical and Computational Fluid Mechanics, $15: 121-141,2001$.

${ }^{27}$ C.H.K. Williamson. 2-D and 3-D aspects of the wake of a cylinder, and their relation to wake computations. In C.R. Anderson and C. Greengard, editors, Lectures in Applied Mathematics, volume 28, pages 719-751. American Mathematical Society, 1991.

${ }^{28}$ S. Mittal. Computation of 3D flows past circular cylinders of low aspect ratio. Physics of Fluids, 13:177-191, 2001.

${ }^{29}$ R. Mittal and S. Balachander. Effect of three-dimensionality on the lift and drag of nominally two-dimensional cylinders. Physics of Fluids, 7:1841-1865, 1995.

${ }^{30}$ S. Mittal. Effect of a "slip" splitter plate on vortex shedding from a cylinder. Physics of Fluids, 15:817-820, 2003.

${ }^{31}$ S. Mittal and B. Kumar. Flow past a rotating cylinder. Journal of Fluid Mechanics, 476:303$334,2003$.

${ }^{32} \mathrm{G}$. Schewe. On the force fluctuations acting on a circular cylinder in crossflow from subcritical up to transcritical Reynolds numbers. Journal of Fluid Mechanics, 133:265-285, 1983.

${ }^{33} \mathrm{P}$. W. Bearman. On vortex shedding from a circular cylinder in the critical Reynolds number regime. Journal of Fluid Mechanics, 37:577-585, 1969.

${ }^{34}$ R. H. Kraichnan. Inertial ranges in two-dimensional turbulence. Physics of Fluids, 10:14171423, 1967. 
${ }^{35}$ J. Paret and P. Tabeling. Intermittency in the two-dimensional inverse cascade of energy: experimental observations. Physics of Fluids, 10:3126-3136, 1998.

${ }^{36}$ U. Frisch. Turbulence. Cambridge University Press, Cambridge, 1995.

${ }^{37}$ C.R. Doering and J.D. Gibbon. Applied analysis of the Navier-Stokes equations. Cambridge University Press, Cambridge, 1995.

${ }^{38}$ M. Lesieur. Turbulence in Fluids. Kluwer Academic Press, London, 1986.

${ }^{39}$ R.D. Henderson. Details of the drag curve near the onset of vortex shedding. Physics of Fluids, 7:2102-2104, 1995.

${ }^{40}$ C.H.K. Williamson and A. Roshko. Oblique and parallel modes of vortex shedding in the wake of a circular cylinder at low Reynolds numbers. Z. Flugwiss. Weltraumforsch, 14:3846, 1990.

${ }^{41}$ C. Norberg. Effects of Reynolds numbers and a low-intensity freestream turbulence on the flow around a circular cylinder. Technol Publ. 87/2, S-412-96, Chalmers University, Goteborg, Sweden, 1987.

${ }^{42}$ W. C. L. Shih, C. Wang, D. Coles, and A. Roshko. Experiments on flow past rough circular cylinders at large Reynolds numbers. In Proc. 2nd Int. Coll. Bluff Body Aerod. Appl., page 150, Melbourne, Australia, 1992.

${ }^{43}$ A. Roshko. Perspectives on bluff body aerodynamics. Journal of Wind Engineering and Industrial Aerodynamics, 49:79-100, 1993. 


\begin{tabular}{cccccc}
\hline Mesh & nodes & elements & $h_{r}^{1} / D$ & $N_{t}$ & domain size \\
\hline M1 & 11384 & 22654 & $1.5 \times 10^{-2}$ & 64 & $50 D \times 50 D$ \\
M2 & 22403 & 44454 & $2 \times 10^{-5}$ & 300 & $38 D \times 16 D$ \\
M3 & 34613 & 68858 & $5 \times 10^{-3}$ & 320 & $38 D \times 16 D$ \\
M4 & 47011 & 93574 & $2 \times 10^{-5}$ & 400 & $38 D \times 16 D$ \\
M5 & 116166 & 231484 & $2 \times 10^{-6}$ & 800 & $38 D \times 16 D$ \\
\hline
\end{tabular}

Table 1. Flow past a cylinder at various Re: details of the finite elements used. 

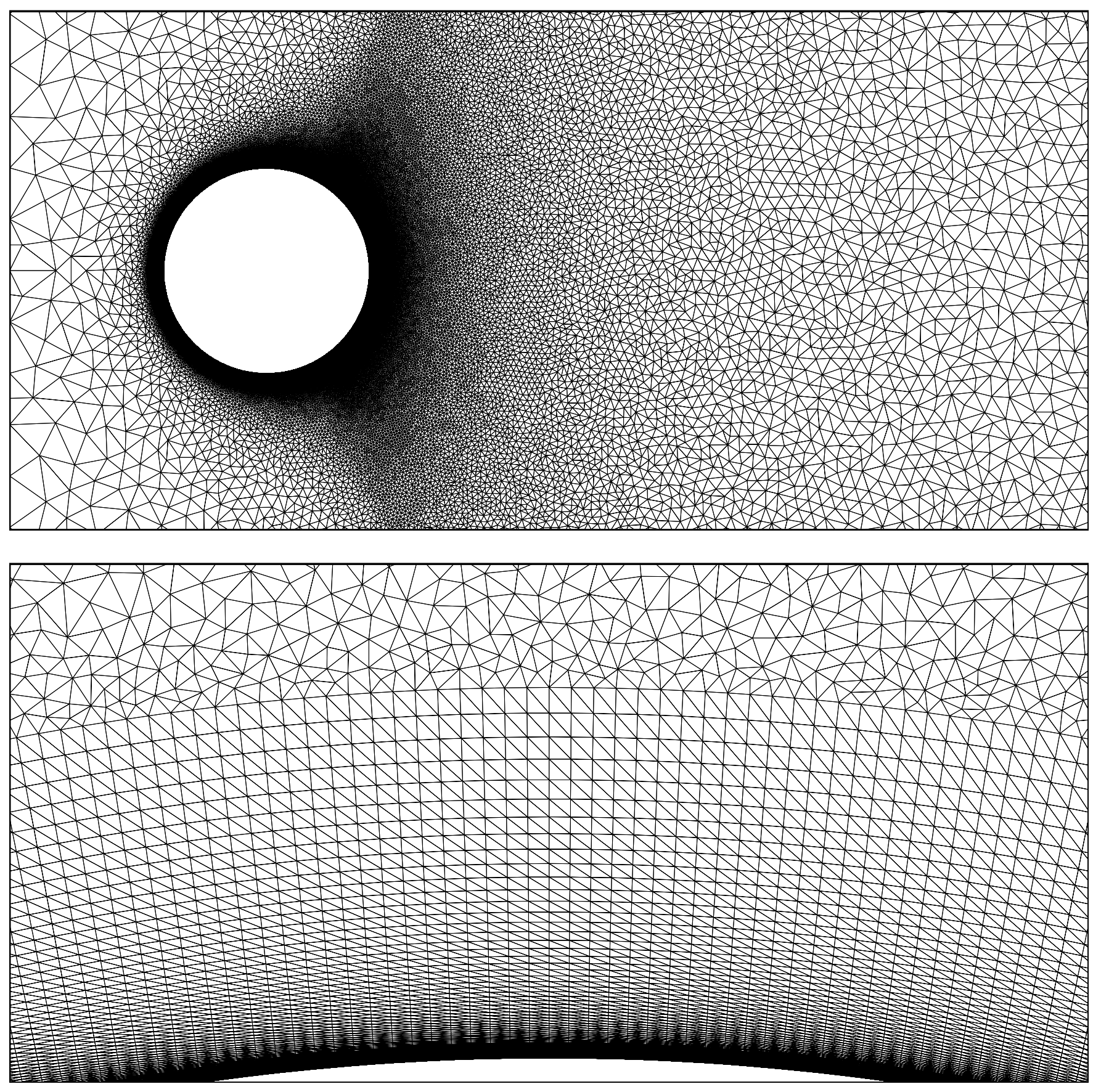

Figure 1: Flow past a cylinder: successive close up views of the finite element mesh M5 with 116, 166 nodes and 231, 484 triangular elements. 

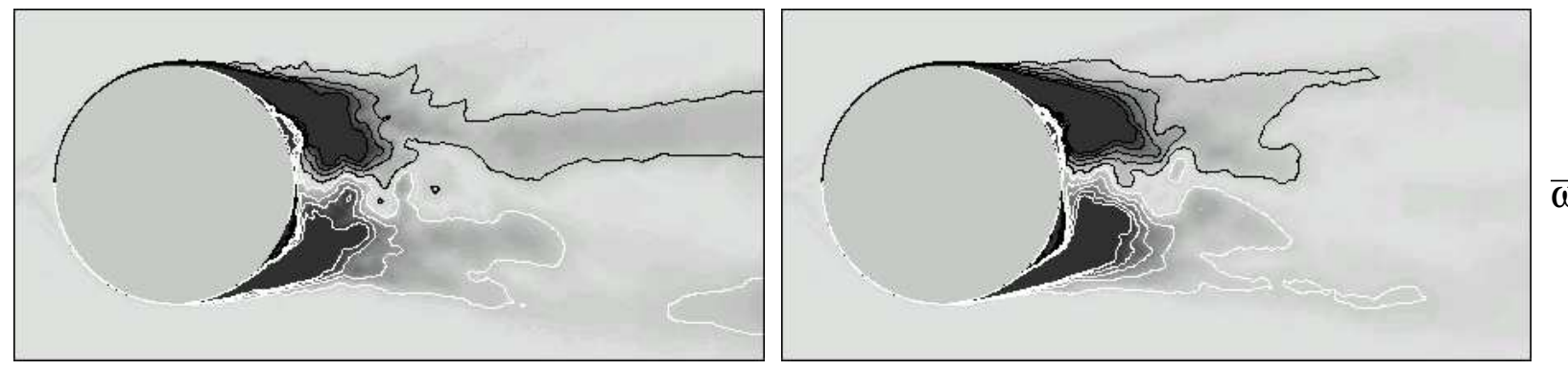

$\bar{\omega}$
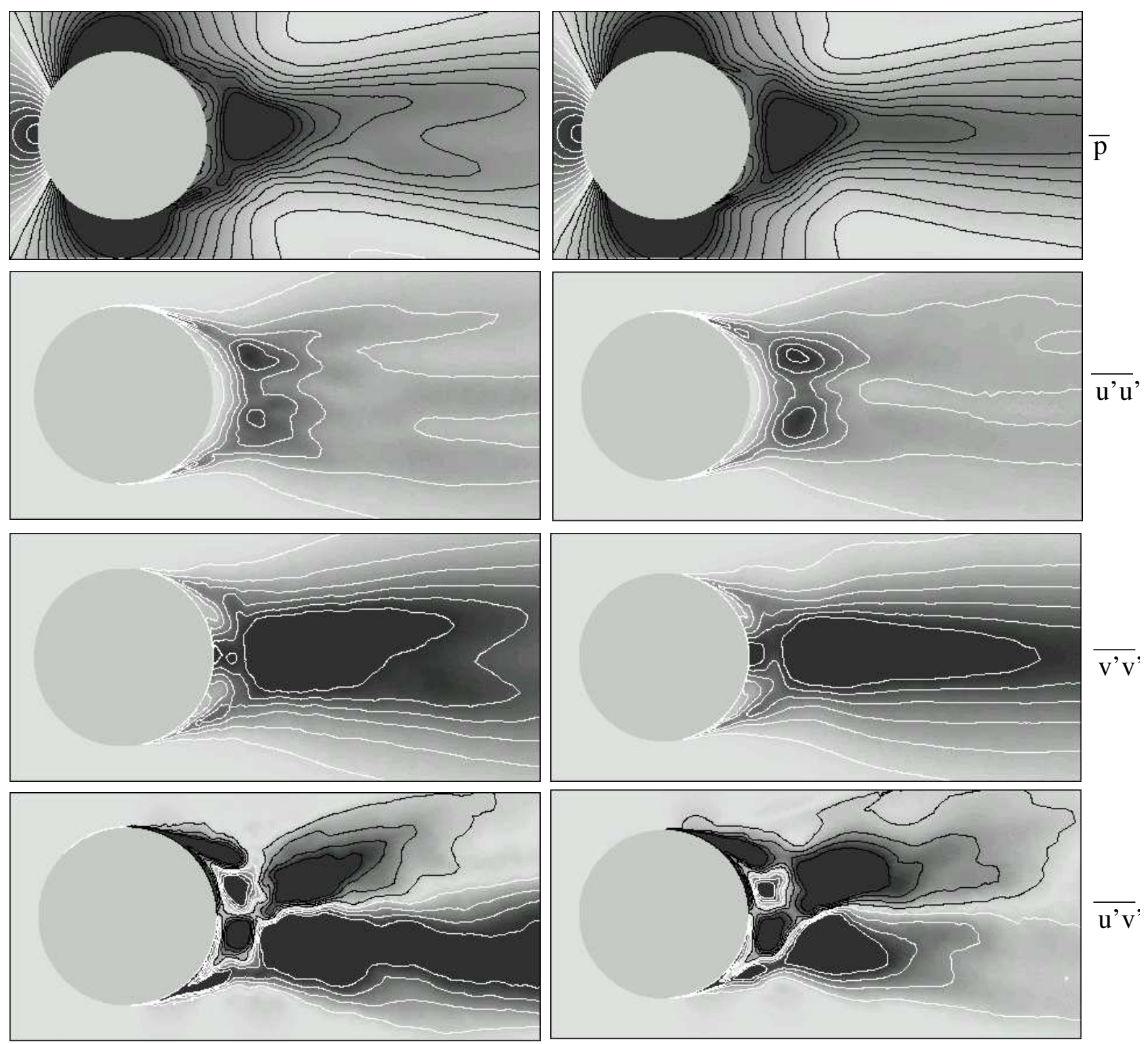

Figure 2: $R e=10^{6}$ flow past a cylinder using mesh $M 4$ : the time averaged vorticity and pressure fields and distribution of Reynolds stresses for 2D LES with a Smagorinsky model (right) and model free (left) calculations. 


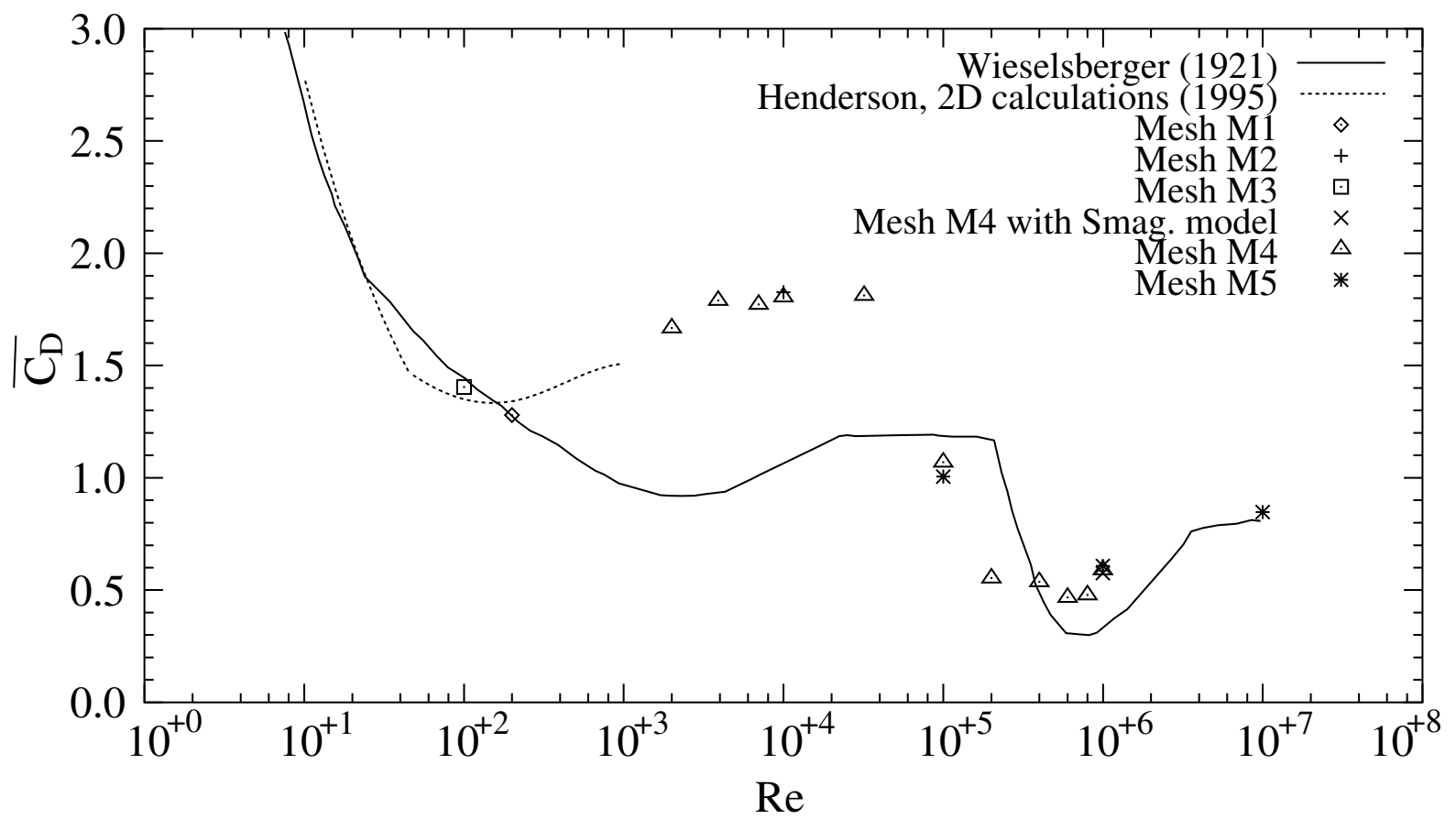

Figure 3: Flow past a cylinder: variation of the time averaged drag coefficient with $R e$. The experimental data from Weiselberger (1921) has been taken from Roshko. ${ }^{10}$ The data from 2D calculations by Henderson ${ }^{39}$ are also shown. 


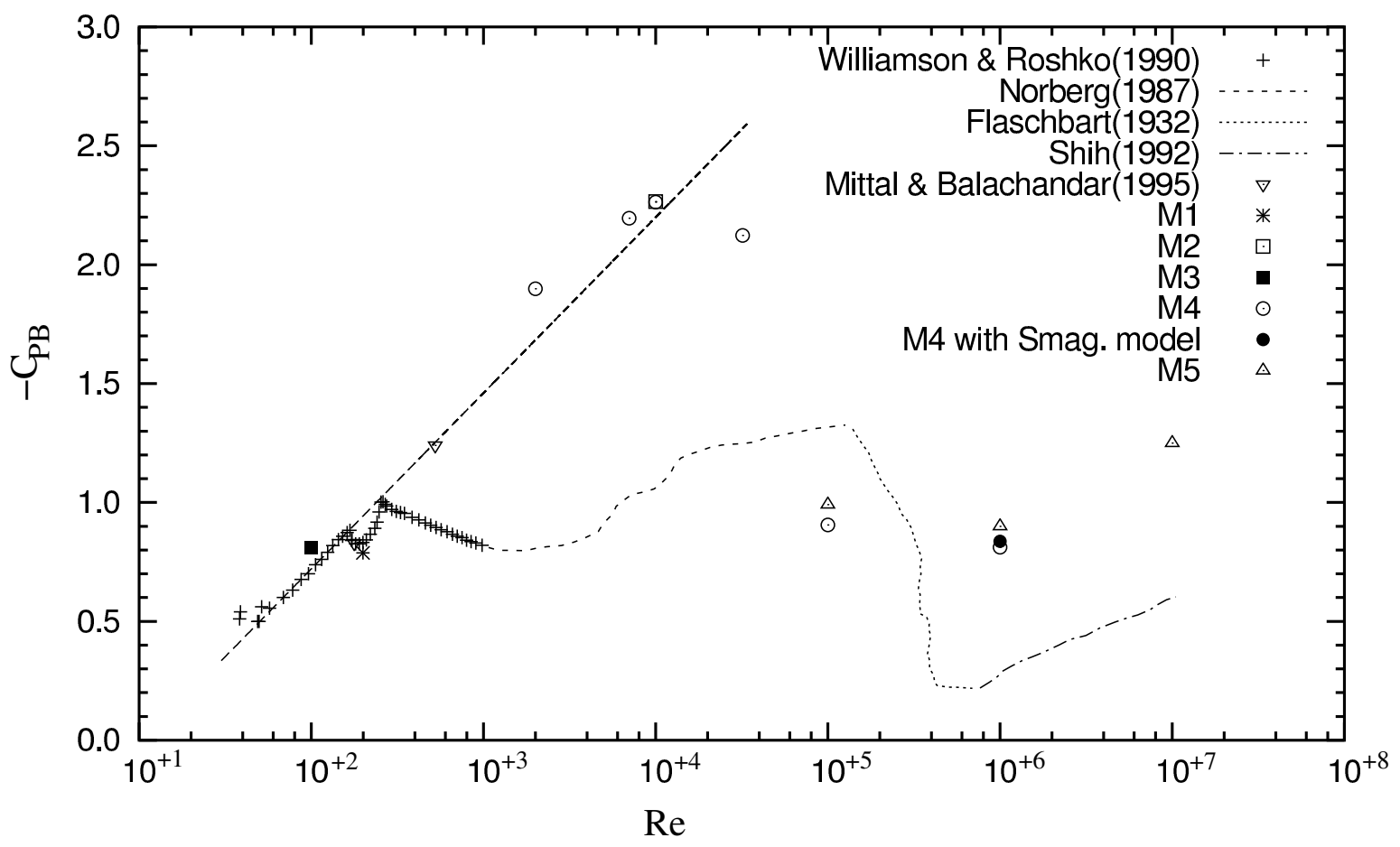

Figure 4: Flow past a cylinder: variation of the time averaged base suction coefficient $\left(-C_{P B}\right)$ with $R e$. The experimental data from various studies by Williamson \& Roshko, ${ }^{40}$ Norberg, ${ }^{41}$ Flaschbart (1992) and Shih et al. ${ }^{42}$ has been taken from Williamson. ${ }^{1}$ 


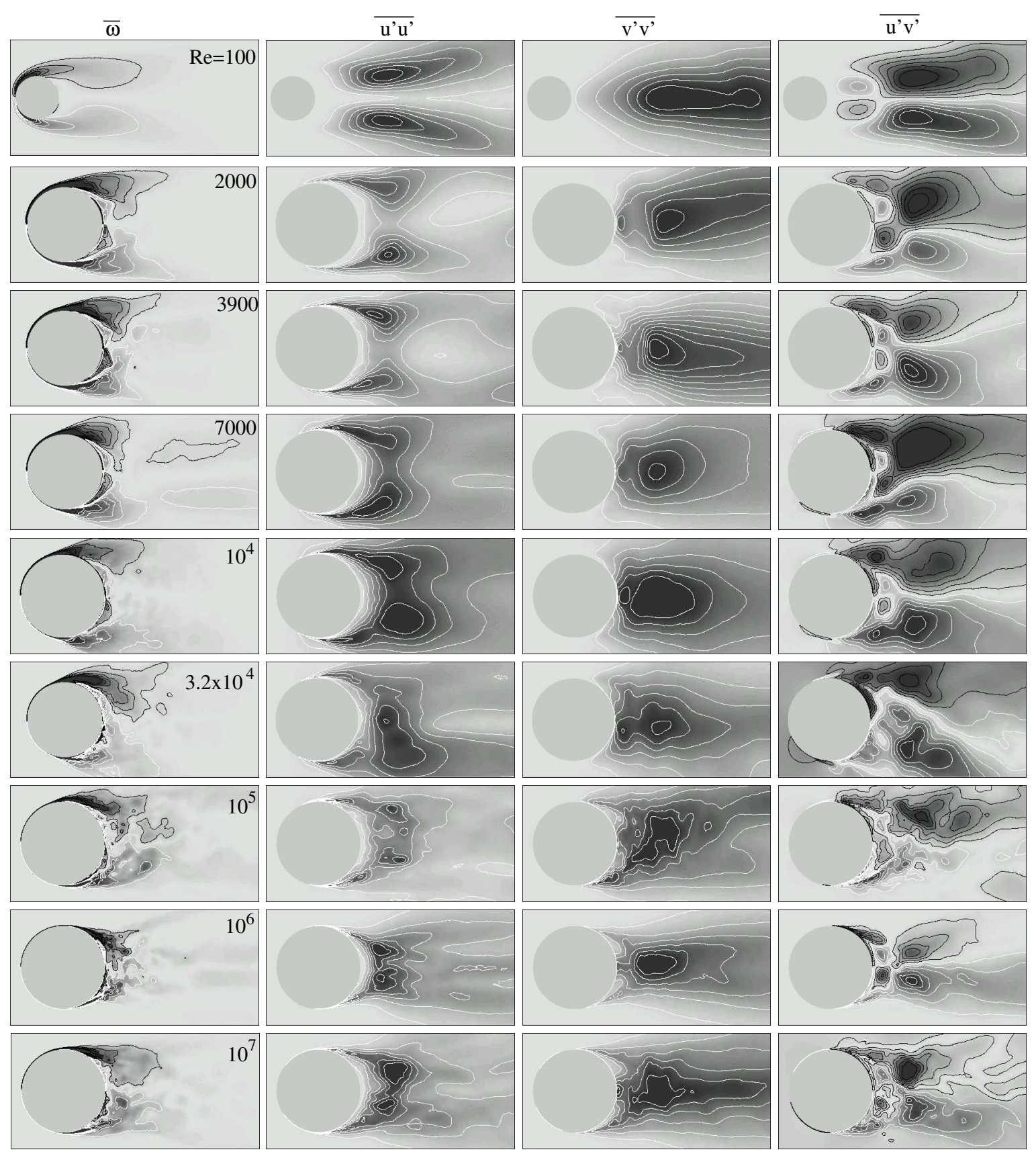

Figure 5: Flow past a cylinder: time averaged vorticity field, and Reynolds stresses $\left(\overline{u^{\prime} u^{\prime}}, \overline{v^{\prime} v^{\prime}}\right.$ and $\overline{u^{\prime} v^{\prime}}$ ) for various $R e$. 

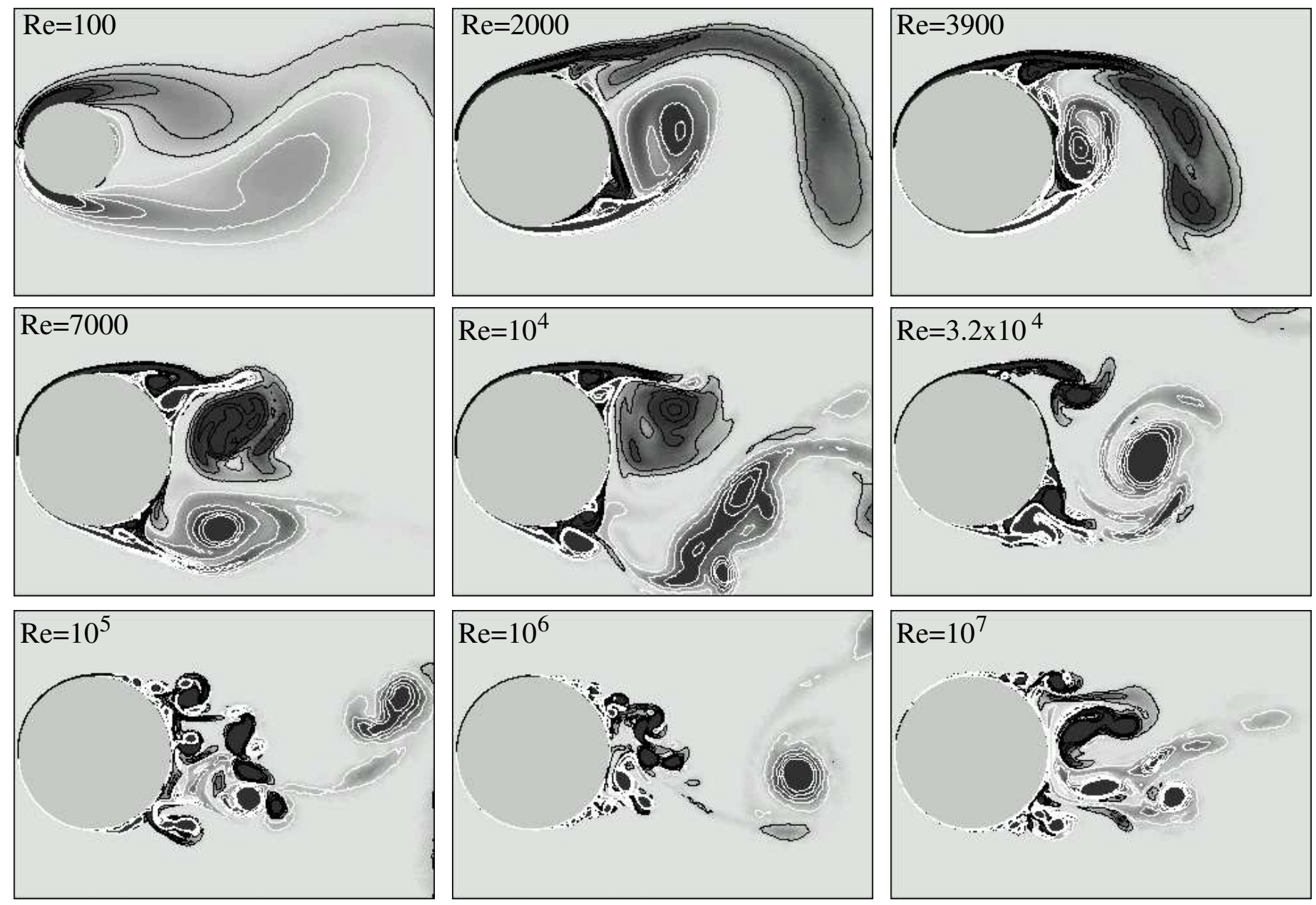

Figure 6: Flow past a cylinder: instantaneous vorticity field for various $R e$. 

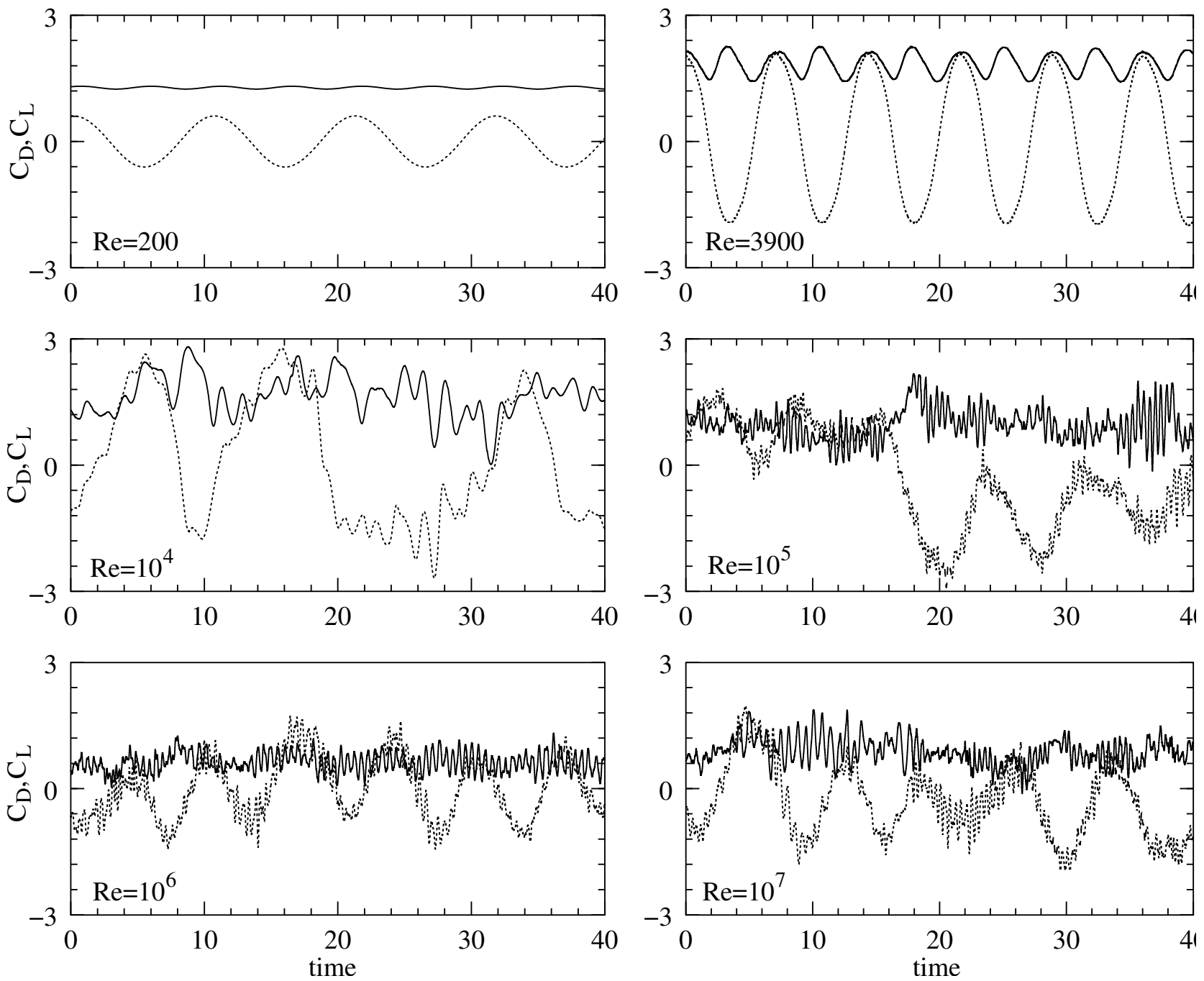

Figure 7: Flow past a cylinder: time histories of drag (solid lines) and lift (broken lines) coefficients for various $R e$. 


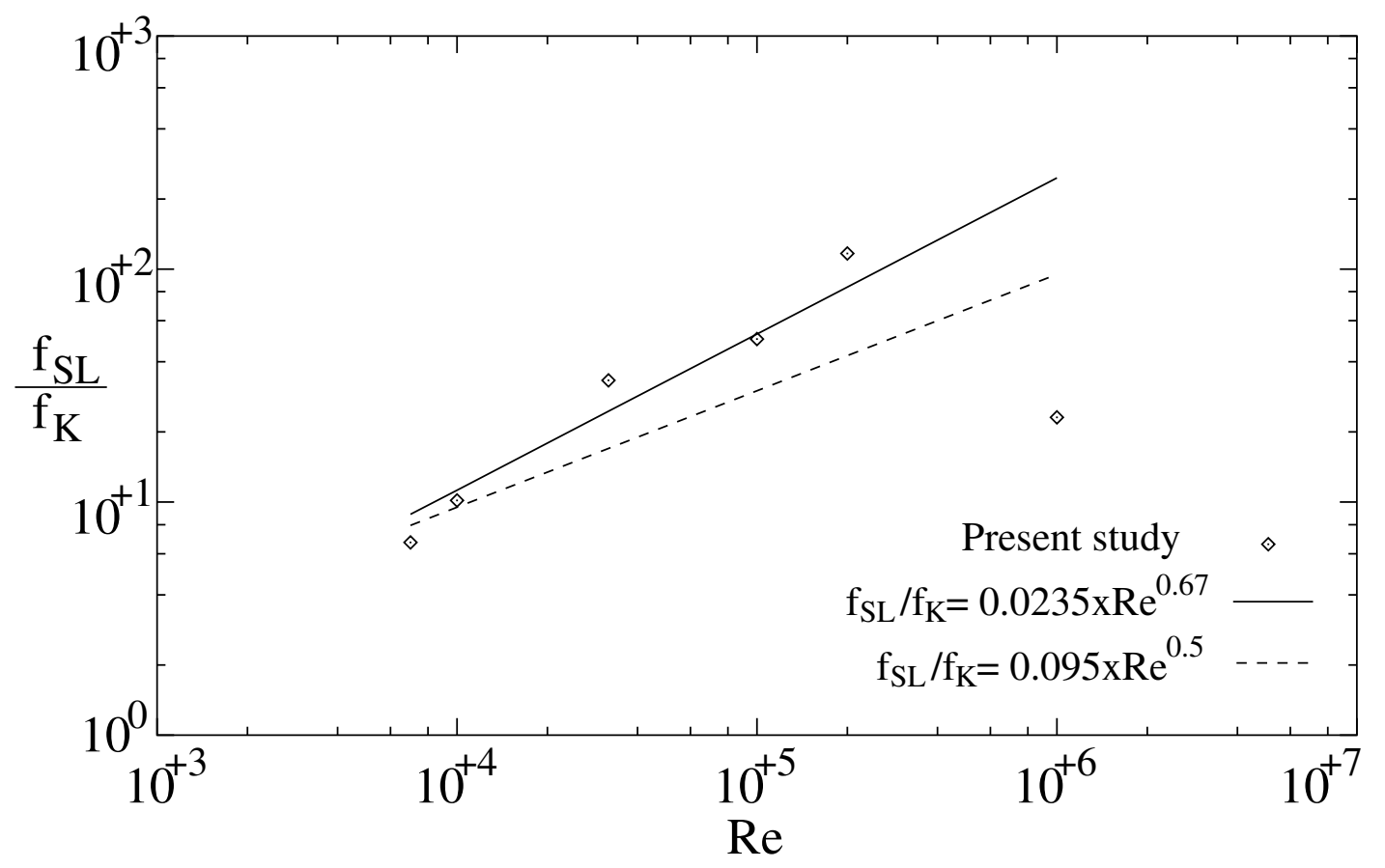

Figure 8: Flow past a cylinder: variation, with $R e$, of the shear layer frequency $\left(f_{S L}\right)$ normalized with vortex shedding frequency $\left(f_{K}\right)$. The expression for the $R e^{0.67}$ power law is from from Prasad and Williamson. ${ }^{8}$ The $R e^{0.5}$ power law is from an approximate analysis. 

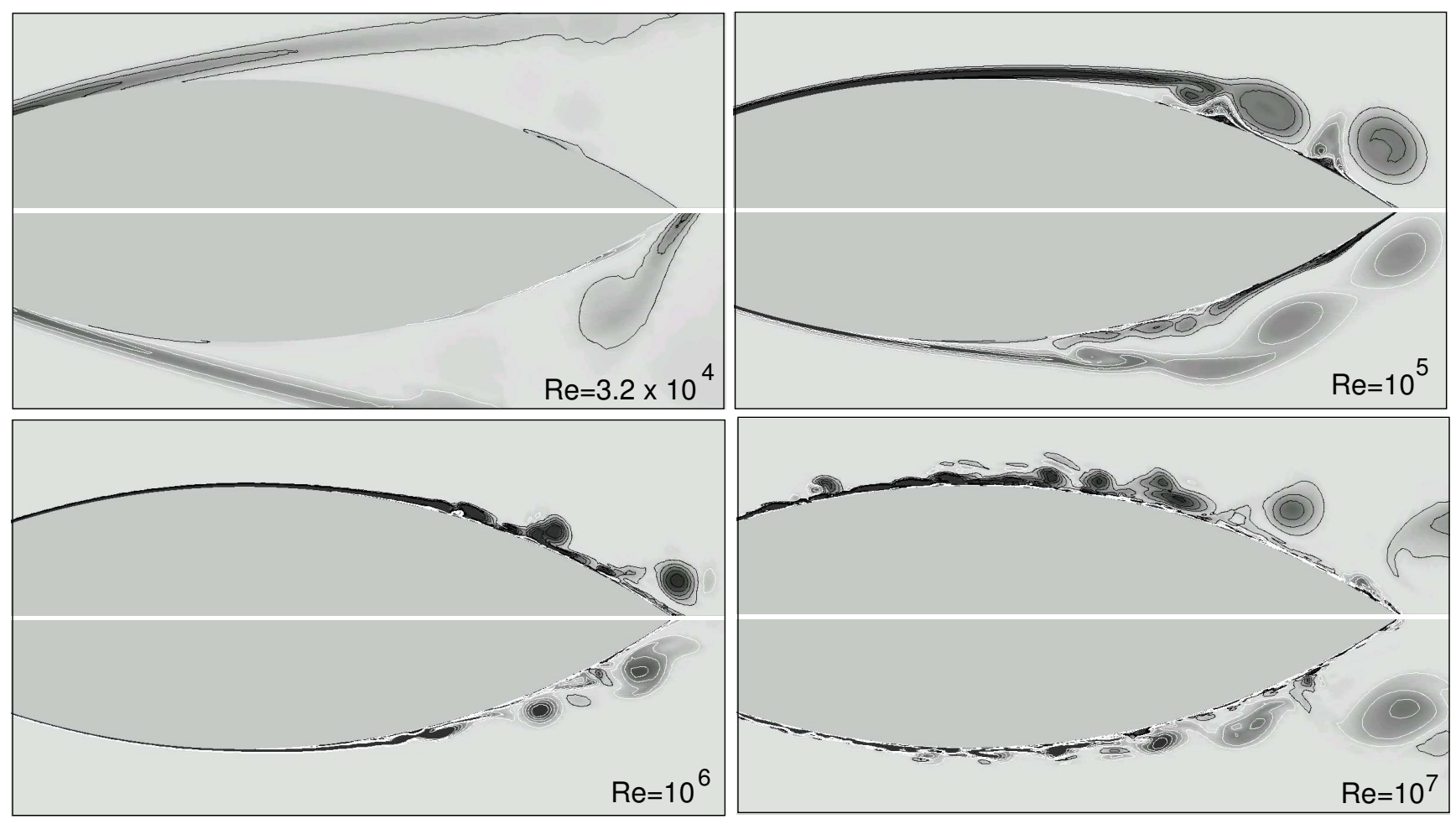

Figure 9: Flow past a cylinder: close-up view, near the upper and lower shoulder of the cylinder, of the instantaneous vorticity field for various $R e$. 


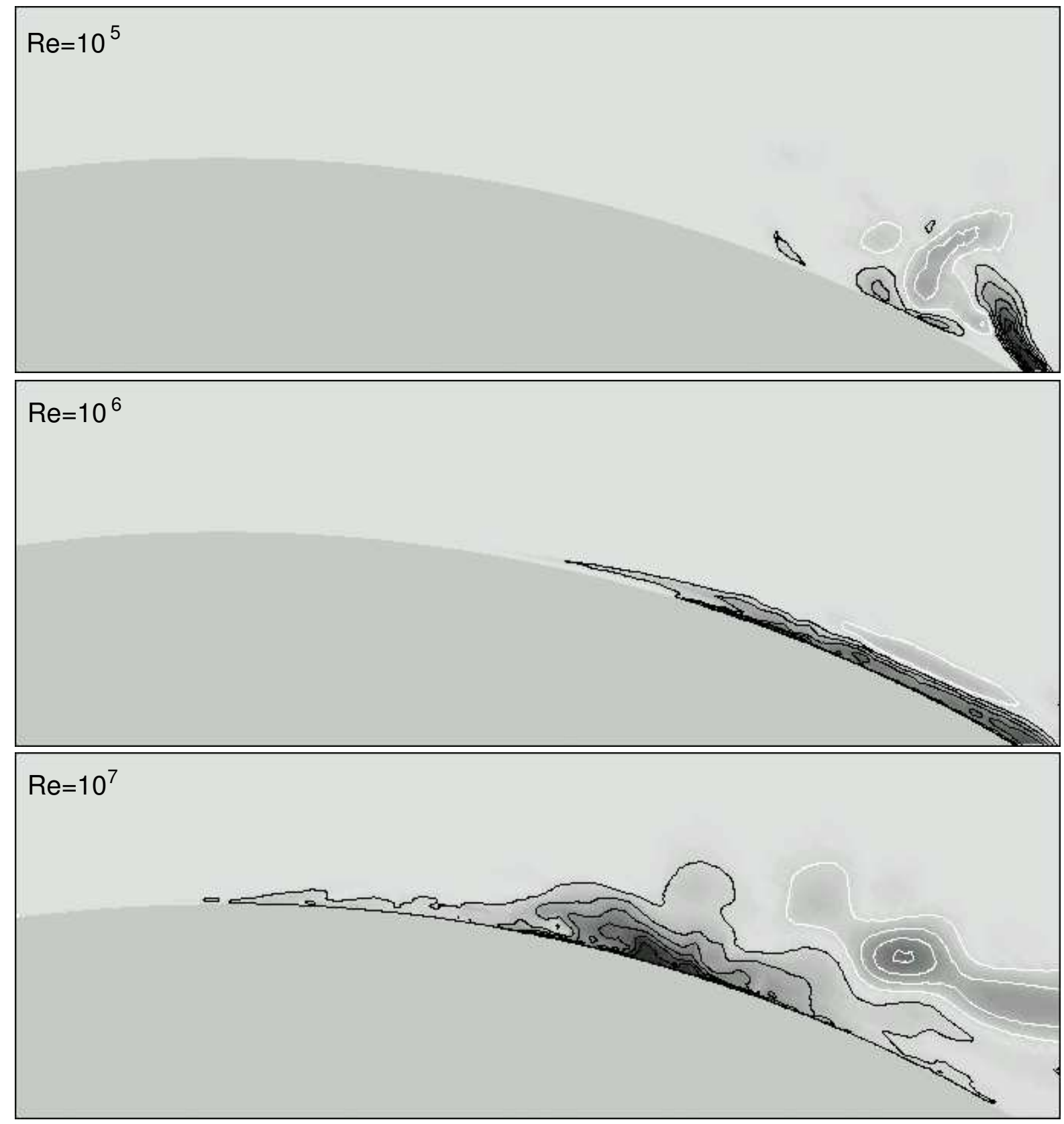

Figure 10: Flow past a cylinder: close-up view of the $\overline{u^{\prime} v^{\prime}}$ distribution. The averaging has been done for one shear layer cycle. 


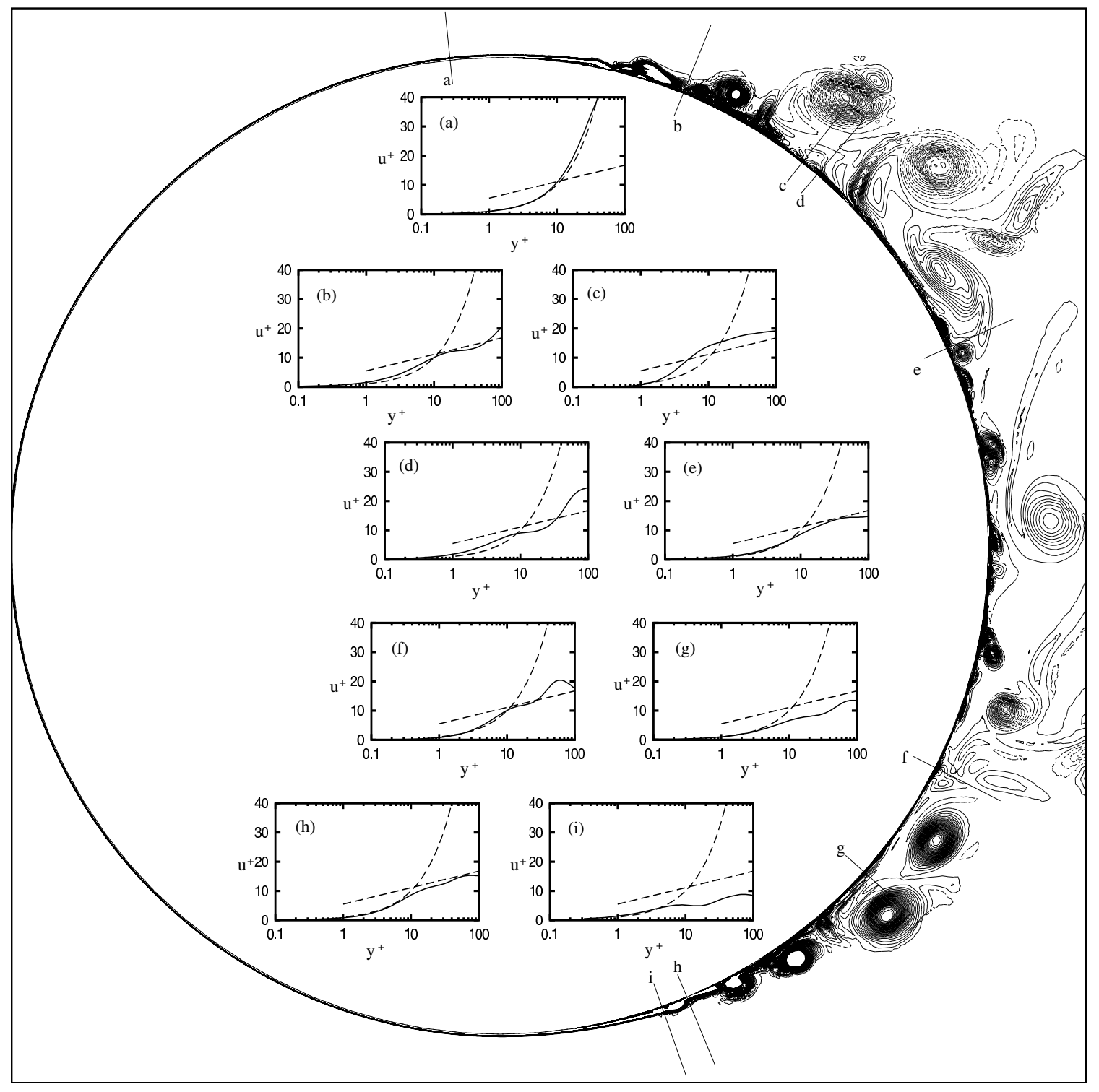

Figure 11: $R e=10^{6}$ flow past a cylinder: instantaneous vorticity field and velocity profiles at various stations in wall co-coordinates. Positive vorticity is shown in solid lines and negative in broken lines. Also shown in broken lines is the velocity profile in the viscous sublayer and log layer in a turbulent boundary layer on a flat plate. 


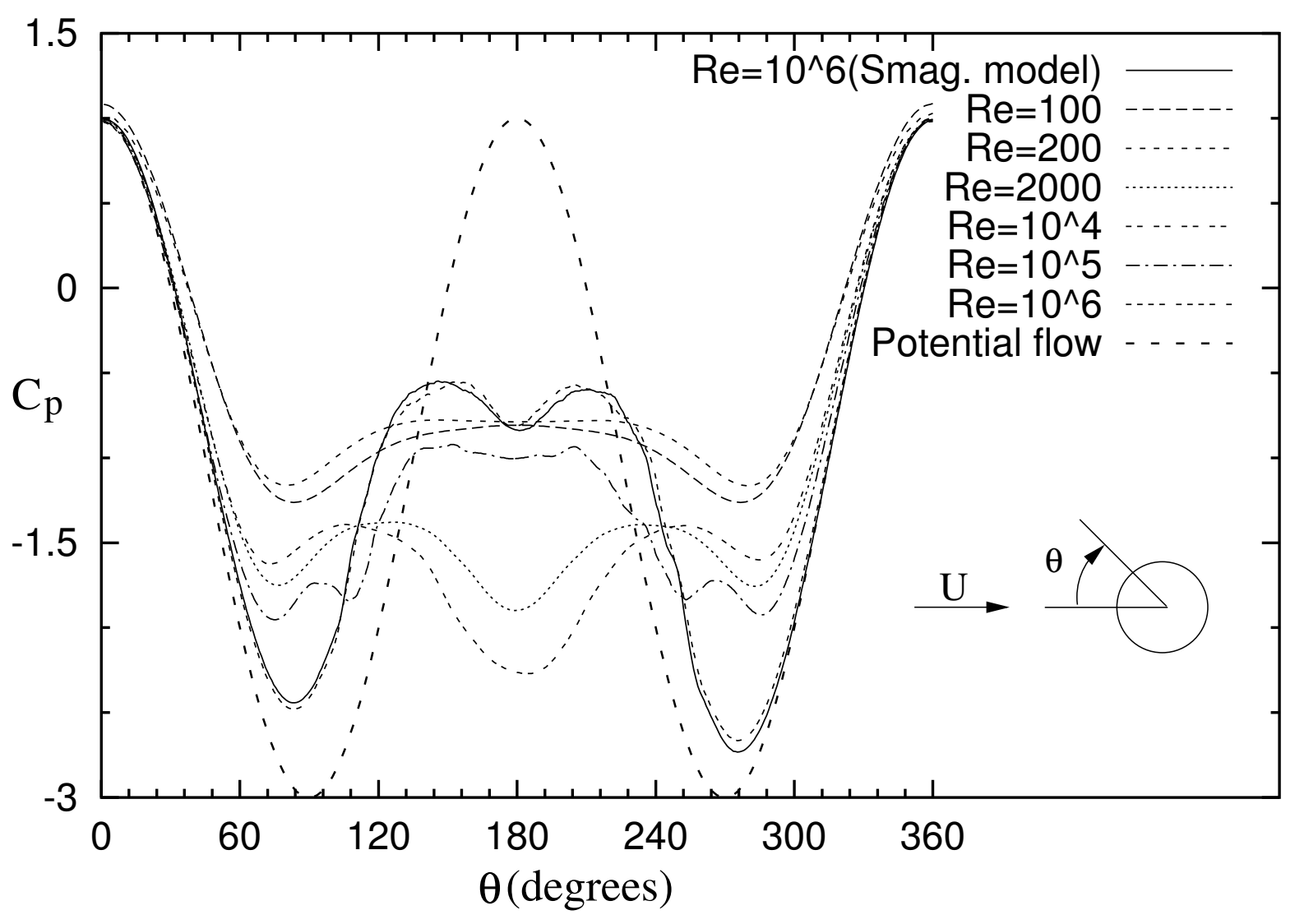

Figure 12: Flow past a cylinder: pressure distribution on the surface of the cylinder for the time averaged flow field. 

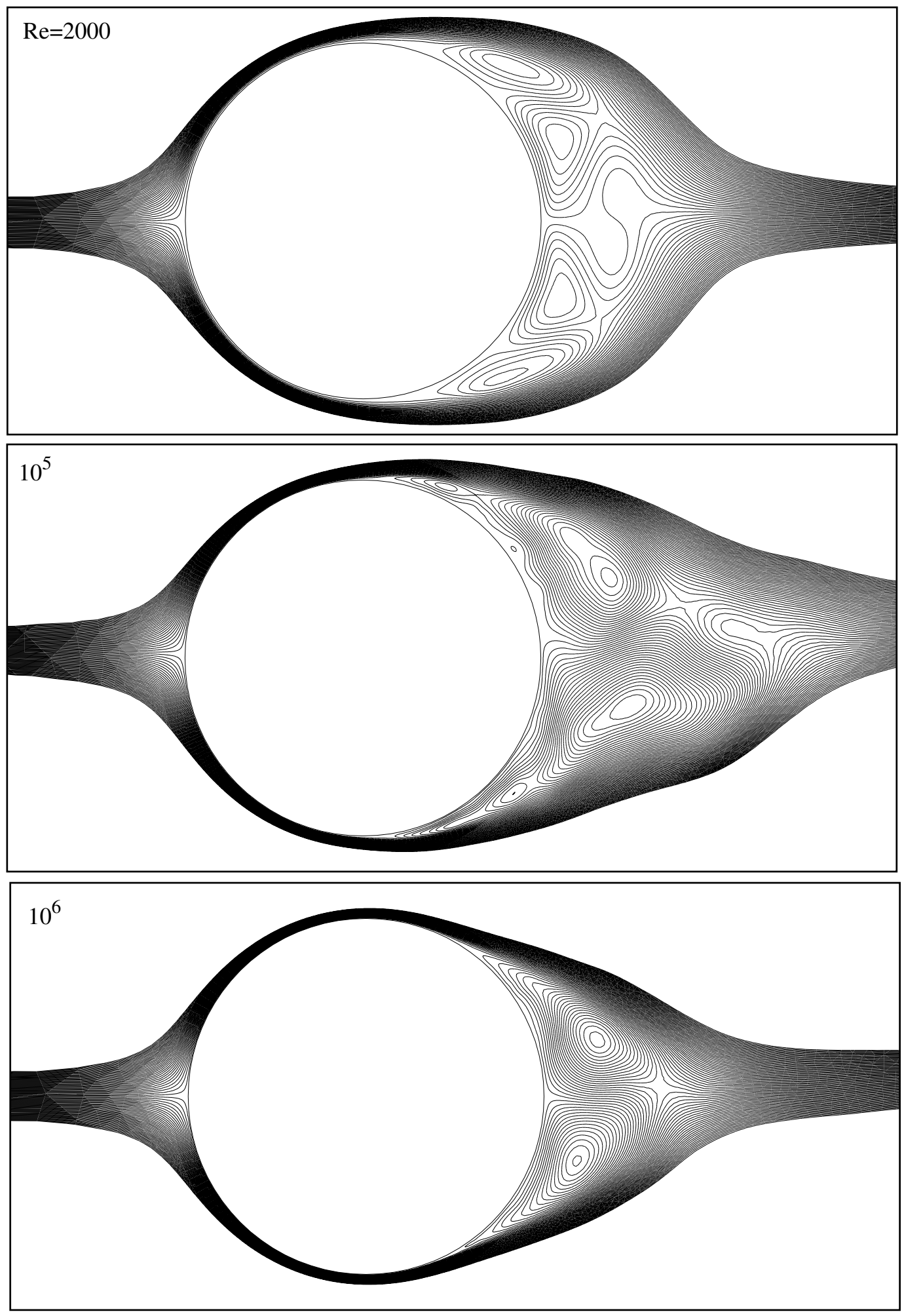

Figure 13: Flow past a cylinder: streamlines for the time averaged flow field for $R e=2000$, $10^{5}$ and $10^{6}$. 


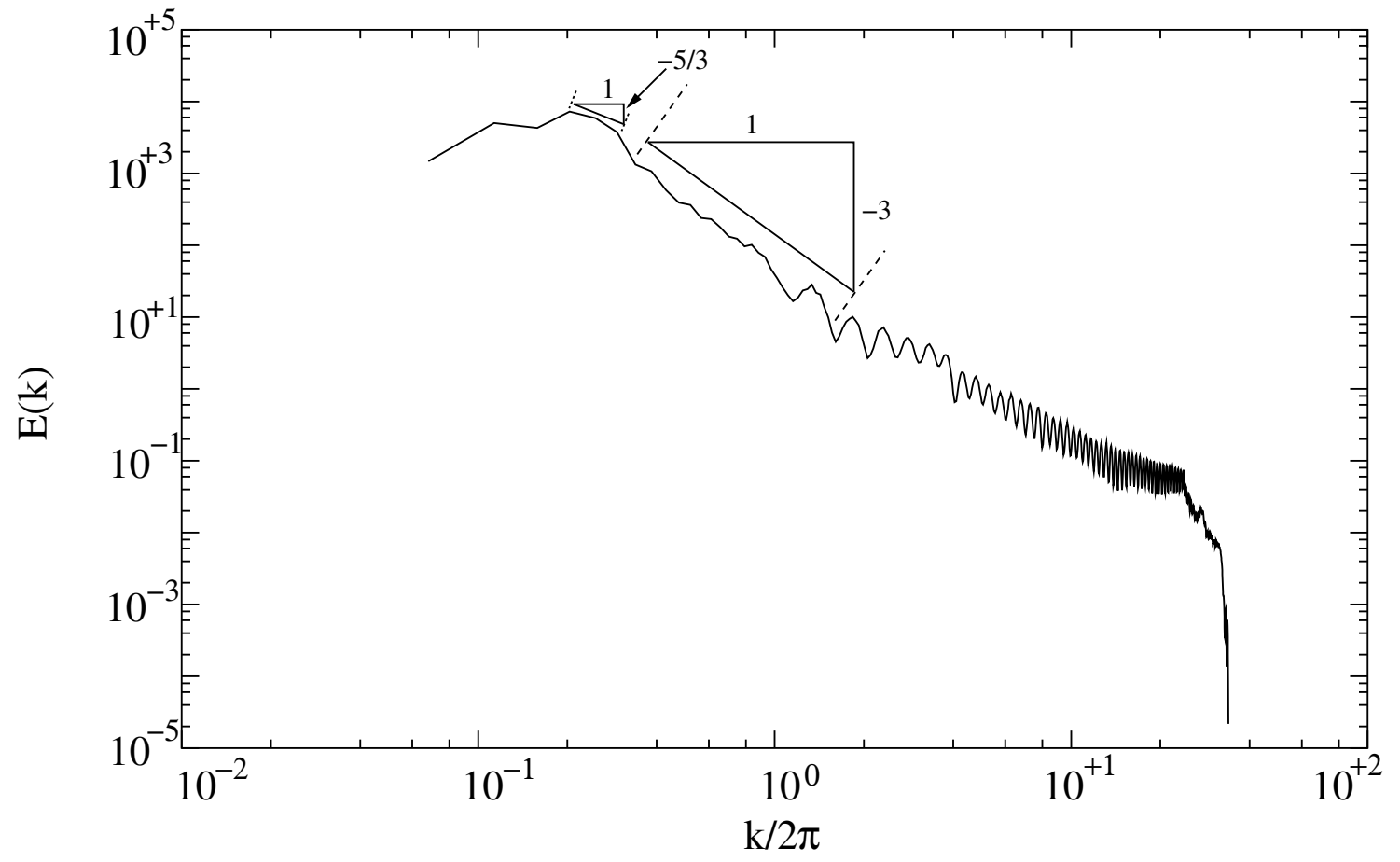

Figure 14: $R e=10^{6}$ flow past a cylinder with mesh $M 5$ : spectrum of the spatial distribution of energy. 


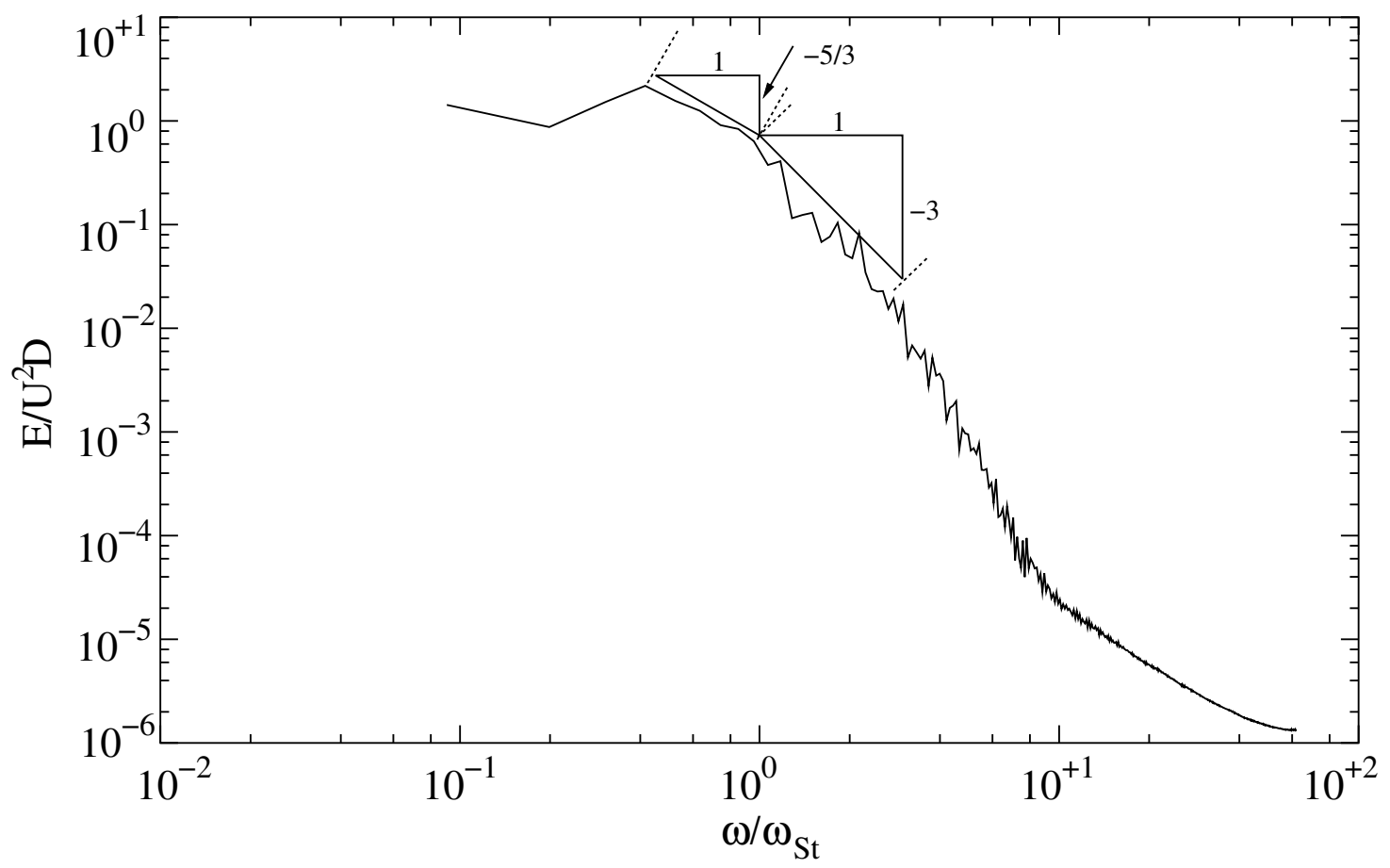

Figure 15: $R e=10^{6}$ flow past a cylinder with mesh $M 5$ : spectrum of the temporal variation of energy sampled at $(x / D=0.26, y / D=-0.46)$. 
Figure 1: Flow past a cylinder: successive close up views of the finite element mesh M5 with 116, 166 nodes and 231, 484 triangular elements.

Figure 2: $R e=10^{6}$ flow past a cylinder using mesh $M 4$ : the time averaged vorticity and pressure fields and distribution of Reynolds stresses for 2D LES with a Smagorinsky model (right) and model free (left) calculations.

Figure 3: Flow past a cylinder: variation of the time averaged drag coefficient with Re. The experimental data from Weiselberger (1921) has been taken from Roshko. ${ }^{10}$

Figure 4: Flow past a cylinder: variation of the time averaged base suction coefficient $\left(-C_{P B}\right)$ with Re. The experimental data shown is from Williamson \& Roshko, ${ }^{40}$ Norberg, ${ }^{41}$ Flaschbart (1992) taken from Roshko ${ }^{43}$ and Shih et al.. ${ }^{42}$

Figure 5: Flow past a cylinder: time averaged vorticity field, and Reynolds stresses $\left(\overline{u^{\prime} u^{\prime}}, \overline{v^{\prime} v^{\prime}}\right.$ and $\overline{u^{\prime} v^{\prime}}$ ) for various $R e$.

Figure 6: Flow past a cylinder: instantaneous vorticity field for various Re.

Figure 7: Flow past a cylinder: time histories of drag (solid lines) and lift (broken lines) coefficients for various $R e$.

Figure 8: Flow past a cylinder: variation, with $R e$, of the shear layer frequency $\left(f_{S L}\right)$ normalized with vortex shedding frequency $\left(f_{K}\right)$. The expressions for the $R e^{0.67}$ and $R e^{0.5}$ power laws are from Prasad and Williamson ${ }^{8}$ and Kourta et al., ${ }^{5}$ respectively.

Figure 9: Flow past a cylinder: close-up view, near the upper and lower shoulder of the cylinder, of the instantaneous vorticity field for various $R e$.

Figure 10: Flow past a cylinder: close-up view of the $\overline{u^{\prime} v^{\prime}}$ distribution. The averaging has been done for one shear layer cycle.

Figure 11: $R e=10^{6}$ flow past a cylinder: instantaneous vorticity field and velocity profiles at various stations in wall co-coordinates. Positive vorticity is shown in solid lines and negative in broken lines. Also shown in broken lines is the velocity profile in the viscous sublayer and log layer in a turbulent boundary layer on a flat plate.

Figure 12: Flow past a cylinder: pressure distribution on the surface of the cylinder for the time averaged flow field.

Figure 13: Flow past a cylinder: streamlines for the time averaged flow field for $R e=2000$, $10^{5}$ and $10^{6}$.

Figure 14: $R e=10^{6}$ flow past a cylinder with mesh $M 5$ : spectrum of the spatial distribution of energy. 
Figure 15: $R e=10^{6}$ flow past a cylinder with mesh $M 5$ : spectrum of the temporal variation of energy sampled at $(x / D=0.26, y / D=-0.46)$. 